\title{
The Principal Aspects of Application of Detonation in Propulsion Systems
}

\author{
A. A. Vasil'ev \\ Lavrentyev Institute of Hydrodynamics SB RAS, Novosibirsk 630090, Russia \\ Correspondence should be addressed to A. A. Vasil'ev; gasdet@hydro.nsc.ru \\ Received 1 October 2012; Revised 10 March 2013; Accepted 15 March 2013 \\ Academic Editor: Eliseo Ranzi
}

Copyright (C) 2013 A. A. Vasilev. This is an open access article distributed under the Creative Commons Attribution License, which permits unrestricted use, distribution, and reproduction in any medium, provided the original work is properly cited.

\begin{abstract}
The basic problems of application of detonation process in propulsion systems with impulse and continuous burning of combustible mixture are discussed. The results on propagation of detonation waves in supersonic flow are analyzed relatively to air-breathing engine. The experimental results are presented showing the basic possibility of creation of an engine with exterior detonation burning. The base results on optimization of initiation in impulse detonation engine are explained at the expense of spatial and temporal redistribution of an energy, entered into a mixture. The method and technique for construction of highly effective accelerators for deflagration to detonation transition are discussed also.
\end{abstract}

\section{Introduction}

The increased interest to use of a detonation process in various technological devices and, in particular, at development of the concept of detonation engine (DE) is stipulated by classical conclusion (e.g., [1]) that from every possible mode of burning of a combustible mixture, the regime of self-sustained detonation (the ideal Chapman-Jouguet wave) is characterized by minimum irreversible losses. The point D (Figure 1) of a tangency of the Michelson-Rayleigh line ODS to a detonation branch 1 of adiabatic curve of energy release $Q=$ const corresponds to minimum growth of an entropy $\Delta S_{D}=\min$ (isentropic curve is tangent to an adiabatic curve from below) in a comparison with any other points. The higher losses are inherent for combustion modes (laminar and turbulent) on a comparison with the C-J detonation mode: the point $F$ of a tangency of the MichelsonRayleigh line OF to lower deflagration branch corresponds to maximum growth of an entropy (in $F$ isentropic curve is tangent to an adiabatic curve from above: $\Delta S_{F}=\max$ ). $\Delta S_{D}=$ min is the first advantage of detonation mode.

The second advantage is the maximum high pressure of a detonation products $P_{D}$ on a comparison with traditional combustion, where a final condition is close to point $P_{P}$ (to be in general agreement with condition $P=$ const) or to point $P_{V}$ (burning at condition $V=$ const).

Only these two advantages of detonation burning allow to get positive profit at consequent expansion of DW-products.

Additional advantage connects with huge velocities of a mixture burning in a detonation wave (DW) and the highest power (energy in unit time) of detonation energy-release, unattainable for combustion conditions.

\section{The Basic Scheme of Detonation Engines}

To the present time for a realization of idea of burning of a combustible mixture in detonation modes, the set of various devices is offered, including numerous model scheme of a detonation engine (see, e.g., bibliographic lists in the reviews $[2,3])$. In the given work, only the basic scheme is considered:

(a) "weapon" schema of a pulsing detonation engine (PDE);

(b) the schema of supersonic pulsing detonation ramjet engine (PDRJE);

(c) the schema of a mixture burning with the help of steady rotating detonation wave. 
(A) In the traditional "weapon" schema of an impulse engine, a basic element is the rectilinear tube with one closed and one open pipe ends. The tube is filled periodically by a combustible mixture (as a rule, with separate injection of fuel and oxidizer and their consequent mixture). After filling a tube in mixture near the closed pipe end, the initiation of a detonation wave is carried out, then DW propagates along a tube to an open pipe end. After DW exits from a tube, the phase of an outflow of a detonation products occurs (while the pressure of products in a tube will not decrease to a level permitting to begin a new cycle of filling of a tube by freshen combustible mixture). Thus the tractive force (for a unit shot) is ensured at the expense of action of the raised pressure of a detonation products on the closed pipe end (from the moment of DW initiation near the closed pipe end and during consequent DW distribution along a pipe), and action of flowing out of a detonation products (through an open pipe end in a consequent time interval after DW exits from a pipe). From aforesaid it follows, that if the DW near the closed pipe end is not excited (e.g., insufficient powerful initiator), then it at once will reduce the propulsion performance characteristics. The initiation of a detonation is the one of basic aspects of effectiveness of PDE (alongside with problems of mixture, cooling, etc.).

The frequency of shots is determined by summarized time of tube filling by freshen combustible mixture and time of an outflow of a detonation products. The time of an outflow varies insignificantly at fixed geometry of a tube: in general, it is determined by a sound velocity in reaction products, which in turn insignificantly differs for main conditions of a mixture burning. On Figure 2, the values of a sound velocity in products for typical combustion processes of heptane (as model fuel for kerosene) air mixtures are submitted (detonation: $c_{D}, V=$ const: $c_{V}, P=$ const: $c_{P}$, deflagration burning with a maximum velocity: $c_{F}$, initial: $c$ ). Let us mark, that at shifting along an adiabatic energy-release curve from a detonation point downwards to deflagration point the sound velocity is increased. The time of tube filling is the basic time of a unit cycle of detonation device at traditional injection of freshen mixture from the closed pipe end along tube axes. The filling time is considerably reduced at alternative injection of freshen mixtures into tube: perpendicularly to its axes and simultaneously along whole tube length. However, any information was not possible to find about an engineering realization of the similar schema in the literature of last years. At radial injection of freshen mixture the time of DWproduct outflow became as basic time, so the frequency of PDE operation can be increased notably.

Similar schema is used successfully in LIH SB RAS during the latest 30 years in impulse detonation devices for acceleration of micro particles by DW (Figure 3, diameter $20 \mathrm{~mm}$, length $2000 \mathrm{~mm}$ ) and their consequent drawing on various surfaces (detonation coating) or in impulse device for clearing the technological equipment from dust deposit [2].

Multibarrelled scheme (e.g., system of seven coaxial pipes: 6 peripheral, located in tops of a regular hexagon and one central on an axes) allows to increase total frequency of $\mathrm{PDE}$ at the expense of sequential operation of tubes (e.g., in 4 times on 7-barrelled scheme: by first the central tube

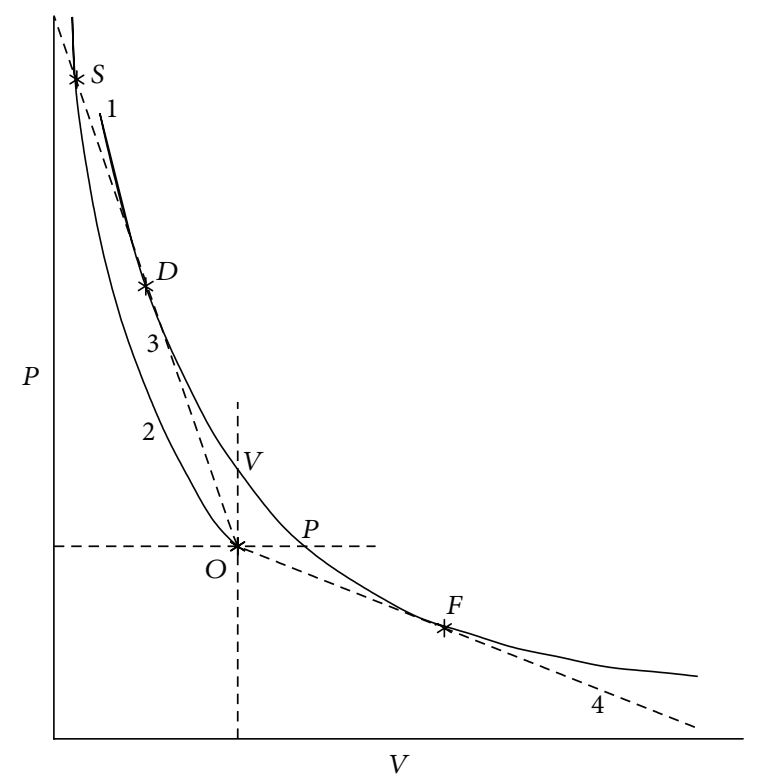

Figure 1: Typical $(P-V)$, diagram of a combustible mixture.

shoots, then peripheral tubes with a defined temporal delay from each other. At this, diametrically located peripheral tubes should work simultaneously). The basic requirement to similar multibarrelled engine is the complete identity of processes in each individual pipe and rigid simultaneity of operation of diametrically pair tubes. Otherwise, it will promote the origin of random rotary moment, operating on an engine. Let us mark, that for identity of impulses, the crosssection of the central tube should twice exceed a cross-section of peripheral tube.

It is necessary to underline that PDE should work cyclically with rather high frequency to compete on effectiveness to a classical engine, in which the stationary combustion is realized. For magnification of tractive force at DW product outflow the open pipe end (usual by whole multibarrelled system) is supplied by additional nozzle.

(B) The schema of supersonic pulsing detonation ramjet engine (PDRJE) represents an alternative type of PDE (Figure 4). The conceptual variant of PDRJE [4] includes an air inlet (index 1 on Figure 4), injection system 2 of fuel in air stream, lengthy combustion chamber 3 , exhausted nozzle 6 , system of mixture initiation 5 (near to exhaust nozzle), and DW 4. DW in the combustion chamber of such engine in an ideal case (in the judgment of the authors [4]) should cyclically be propagated upwards and downwards along a stream at the expense of cyclical enrichment and depletion of a mixture composition. It would be underlined especially that in the combustion chamber the mixture is moved with a supersonic velocity, and instead of the forward closed pipe end, an air inlet is used.

Traditionally detonation is investigated in a motionless mixture. In a case of subsonic or supersonic flows the boundary layer on walls of an aerodynamic tract is an essential singularity of a stream of a combustible mixture. 


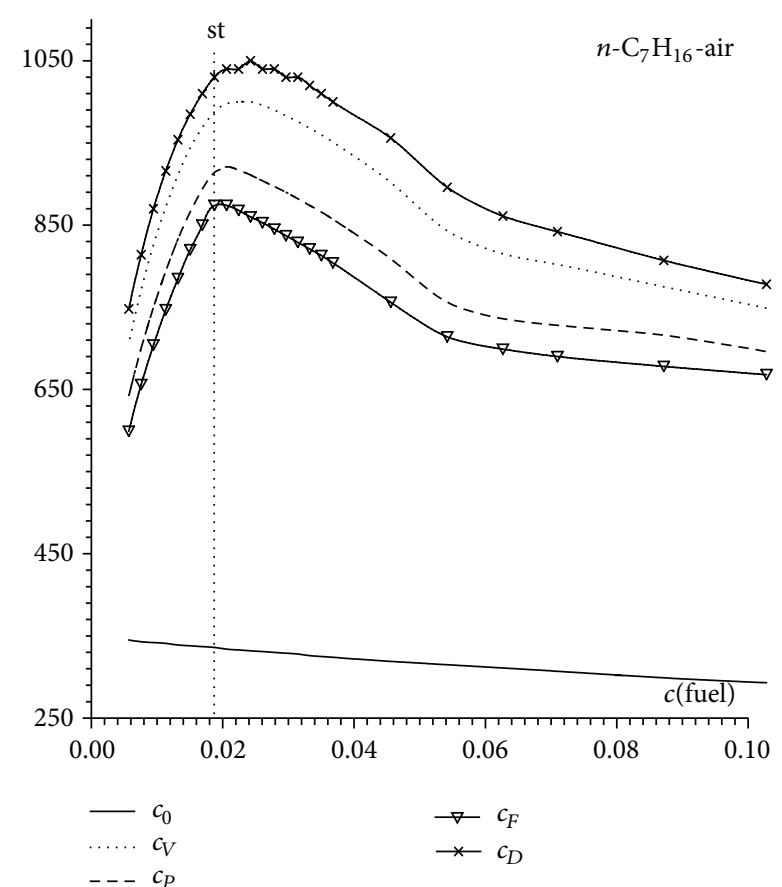

Figure 2: Sound velocities on fuel molar concentration for typical regimes of heptane-air mixtures (indexes correspond to characteristic points on Figure 1, vertical dotted line: stoichiometry).

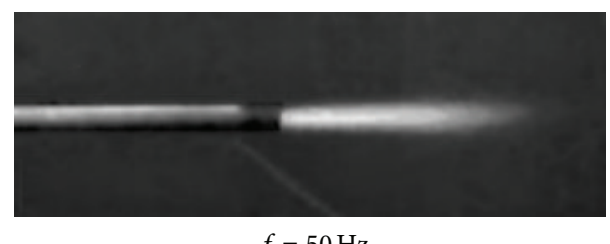

FIGURE 3: Typical photo of operation of pulsed detonation tube of LIH SB RAS with frequency $50 \mathrm{~Hz}$.

As the outcome, gas-dynamic parameters acquire some characteristic profiles instead of homogeneous on cross-section and length of a pipe (that is typical for motionless mixture). For example, on Figure 5 the profiles for a supersonic mixture stream (driving from left to right) are submitted, on which DW is propagated upstream. The laboratory experimental model of offered PDRJE with a single shot is realized by the authors of idea [4], and in [6], some preliminary results of investigation for stream of the Mach number $M=3$ were published (DW initiation near output nozzle and DW distribution upstream). The information about experimental realization of cyclical work of such PDRJE is not known.

In [7] the scheme of PDRJE was used as the prototype with essential distinction: the position of DW-initiation point could vary along an aerodynamic tract. The similar modification is important for an evaluation of possibilities of control on propulsion performance characteristics of a real engine (the constant tractive force of an engine at condition of cruising flight is only one point from a range of necessary modes of engine operations). For a hydrogen-air mixture, a possibility of initiation and propagation of self-sustained

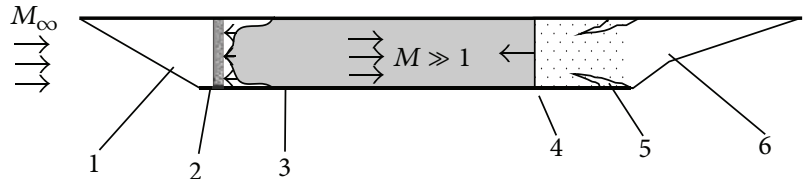

FIGURE 4: Idealized scheme of PDRJE.

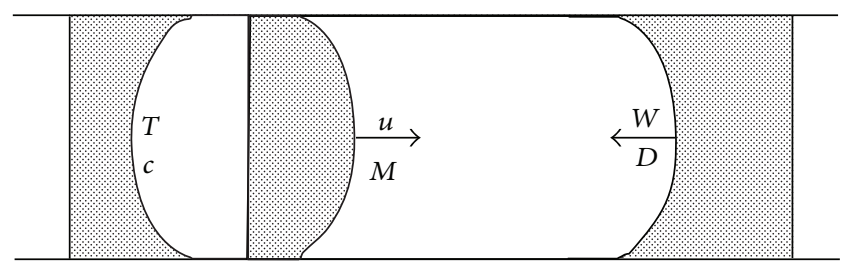

FIGURE 5: Qualitative profiles of gas-dynamic parameters in a supersonic flow of PDRJE.

detonation burning in a supersonic mixture stream of $M=4$ in range of $\alpha=0.5 \div 2.5$ ( $\alpha$ is the excess air factor) was shown experimentally. Thus, is established that the velocity of DW-propagation upstream of a combustible mixture exceeds the C-J velocity for a motionless mixture and is less than it at DW propagation down (Figure 6). A distance, on which the DDT effect was observed (DDT length), equals some channel diameters and approximately twice less in case of DW-distribution upstream. At removal from stoichiometric ratio of fuel-oxidizer to concentration limits the regimes with an unstable detonation and lengthy zone of forming of detonation front were observed. The hypothesis about probable nature of observable effects was expressed permitting qualitatively to explain not only regularities of given work, but also results of another authors.

(C) The schema of a detonation engine with steady rotating $\mathrm{DW}(\mathrm{RDE})$ is original alternative to scheme of pulsing engines.

The essence of idea is the next. In a rectilinear pipe of a round (constant) cross-section at lowering of initial pressure of motionless mixture the regime of stationary DW propagation along pipe axes with the single transversal wave (TW), rotating along an interior concave surface, can be realized (so-called spin detonation). The TW of spinning DW can be stabilized and transformed to rotating wave in some plane (ring schema of mixture burning), if to use a mixture stream with the appropriate velocity (DW stabilization). However it is impossible to realize practically at use of homogeneous mixture, because the stagnation temperature appears much above than the ignition temperature, and mixture will be ignited by uncontrollably near the channel walls. At the same time at separate injection of mixture components (heterogeneous mixture), the given idea can be realized rather simply (on the ring schema), and in this case, DW is automatically stabilized close to injector plane. The given concept was offered and realized in Lavrentyev Institute of Hydrodynamics (LIH SB RAS, Novosibirsk, Russia) almost 55 years back: a mixture burning in steady rotating spinning DW $[8,9]$, schema of RDE. In Figure 7 the idealized schema of a mixture burning in the ring combustion chamber with 


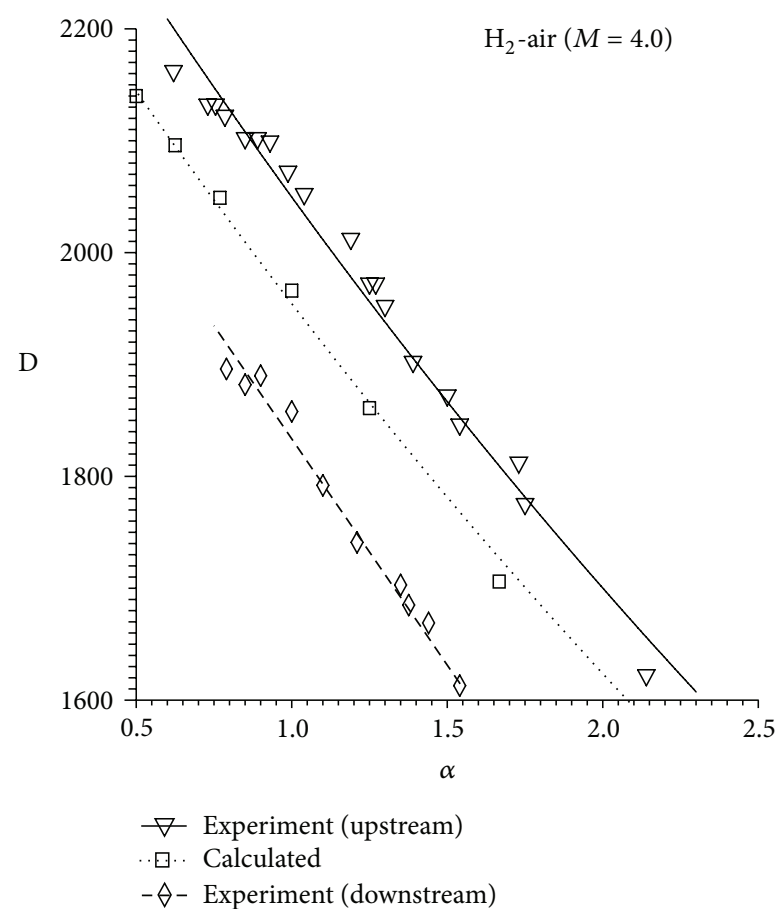

FIGURE 6: DW velocities at propagation upwards (triangles) and downwards (rhombuses) on a supersonic stream of a hydrogenair mixture $(M=4)$ in a comparison with DW velocities for a motionless mixture (small squares) depending on the excess air factor.

the help of two steady rotated DW and typical streak records of a self-luminescence of rotated waves is demonstrated on Figures $7(b)-7(d)$ and Figure 8 (from $[10,11]$ ).

The RDE idea of Professor B. Voitsekhovsky was the leading decision during a long time from a practical point of view. The experimental installation in this period was used for realization of new researches (e.g., $[10,11]$ ) or as an operating experimental model at demonstrating a steady rotating detonation to the delegates of various scientific meetings, for example, to participants of the 2-ICOGERS (Novosibirsk, 1969). In Figure 9 the oscillograms of pressure in steady rotating DW (from [11]) are demonstrated, showing stability of process and its good reproduction (for these investigations the special piezoelectric gauges were used with a filter protecting sensitive piezoceramics from long thermal action of high-temperature detonation products).

The new stage of interest to a rotating detonation has appeared in connection with a "euphoria" termination about fast solution of PDE problem. At beginning of 21 centur it has become obvious that the creation of PDE with effectiveness, exceeding existing engines on stationary combustion, is connected directly with successful solution of a problem of DW initiation (see later). For today LIH is the leader of experimental investigations of a steady rotating detonation (see, e.g., review [9]). Recently, it was possible to realize the rotating DW on fuel-air mixtures [12] for liquid and solid (coal) components.

At use of the DW rotation schema two types of this schema represent a practical interest. First-interchamber burning of a mixture, when the concave walls of the combustion chamber are a natural limit boundary of a mixture. Walls ensure rotary propagation of spinning DW in a fixed crosssection of chamber at the expense of constant overdriven and turn of a stream (left schema on Figure 10 at axial injection of a mixture in combustion chamber. Other modifications are radial injection or injection an angle to an axes).

If in the schema of PDE the pressure on closed pipe end is supposed to be uniformly distributed on end surface and identical in any point, then in the RDE schema the pressure is distributed nonuniformly and the zone with increased pressure rotates together with spinning DW. It is necessary take into account at correct estimation of specific impulse. Once again, it should be underlined that at interchamber burning the concave wall of a pipe plays a major role in a turn of TW and constancy of its axial orientation.

The second schema applies to an exterior burning in detonation mode (right schema in Figure 10), when the layer of reactive mixture is created around a solid cylindrical surface and is burned by a rotating detonation wave. Thus the exterior boundary of a reactive layer appears free (without bounding walls). For a free axially symmetric jet (analog of a gas charge in a pipe) the regime of a spin detonation is not observed: transversal wave of spinning configuration damps without support on the external jet boundary and self-sustaining propagation of DW along jet axes becomes impossible [13]. In such free charge jet the limiting regime of stationary DW propagation along charge axes is typical multifront regime with a great number of TWs on detonation front (instead of spin DW with single TW). The diameter of free gaseous charge for a limiting regime of DW propagation is named traditionally as the critical diameter $d_{*}$ : at $d<d_{*}$; the self-sustaining DW propagation along axes of free charge (without any bounding walls) is not observed. The limiting condition of DW propagation in a pipe with a rigid wall also can be described by "limiting diameter" $d_{\mathrm{lim}}$. It is obvious, that $d_{\lim } \ll d_{*}$.

In un rectilinear pipes the concave rigid wall also plays a basic role in a turn of DW at the expense of a regular and irregular DW reflection and over-driven of a stream near a concave wall. A typical case is a DW turn in a curvilinear branch pipe connecting two rectilinear segments of a detonation pipe.

The problem on a possibility of a mixture burning with maximum rates (schema on Figure 10) in a ring charge with the curvilinear free boundaries has scientific and also practical sense. A minimum three questions arise for a solution of a similar problem: (1) about the physical mechanism of a DW turn; (2) about a minimum radius of a curvature of a ring gas charge; (3) about a minimum thickness of a ring mixture layer, necessary for DW propagation. Really, along a ring charge the propagation of classical DW with radial oriented smooth front (perpendicular to boundary) as in Figure 10 is impossible, because for this purpose the velocity of DW propagation on the exterior boundary of a ring charge should exceed a velocity of DW on the interior boundary despite of a constancy of chemical energy release. It is possible to achieve similar effect only on radial stratified mixtures at careful observance of fuel oxidizer ratio along 

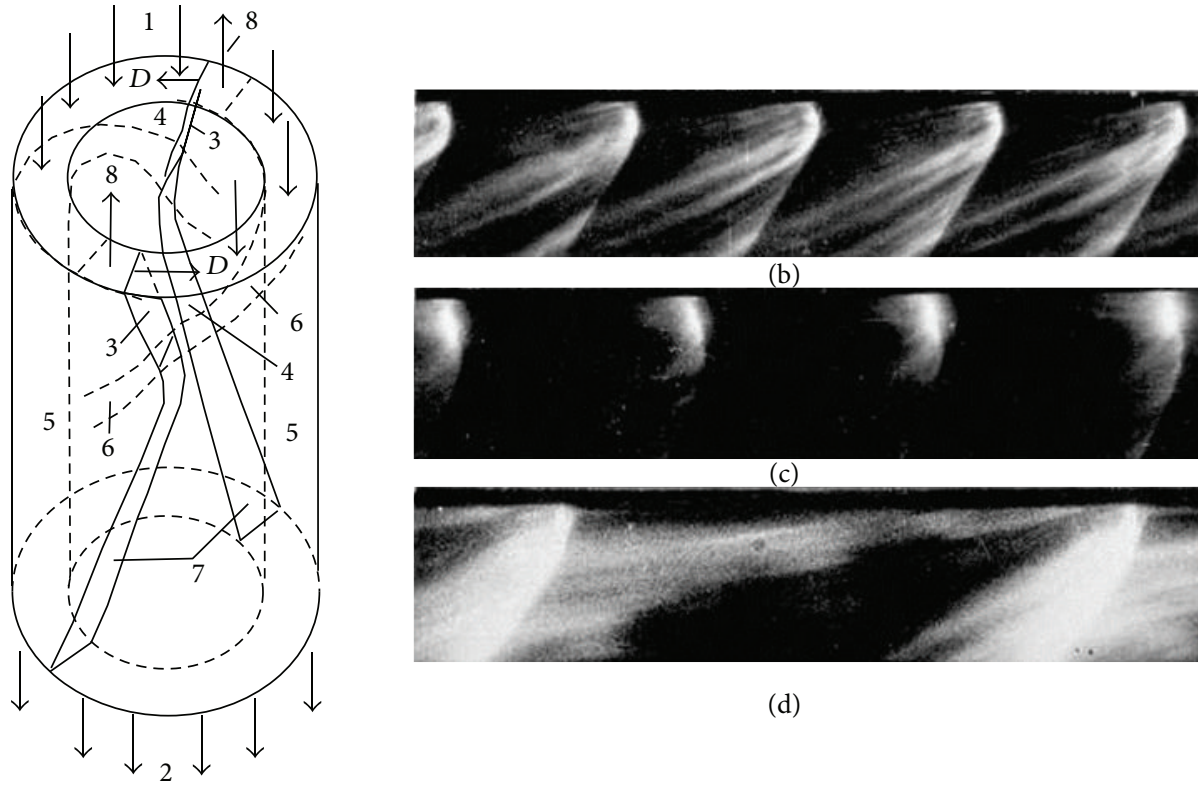

(b)

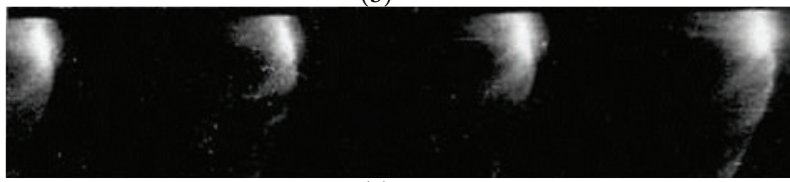

(c)

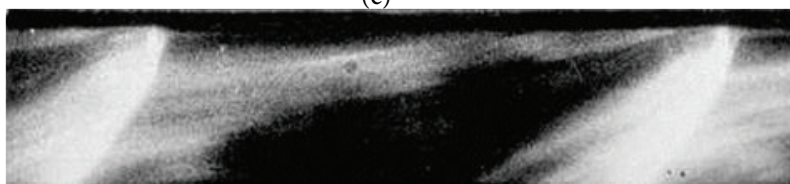

(d)

(a)

FIGURE 7: The schema of continuous burning of a mixture in the flowing ring combustion chamber and typical streak records of self-luminosity of rotating DW at registration across slit paralleled to axis.

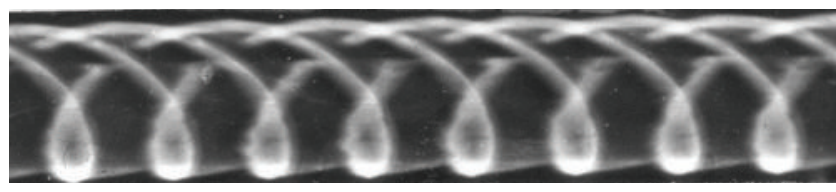

FIGURE 8: Typical streak records of self-luminosity of steady rotating DWs in circle combustion channel at registration along axis (radial mixture flow, three rotating waves on channel circuit).
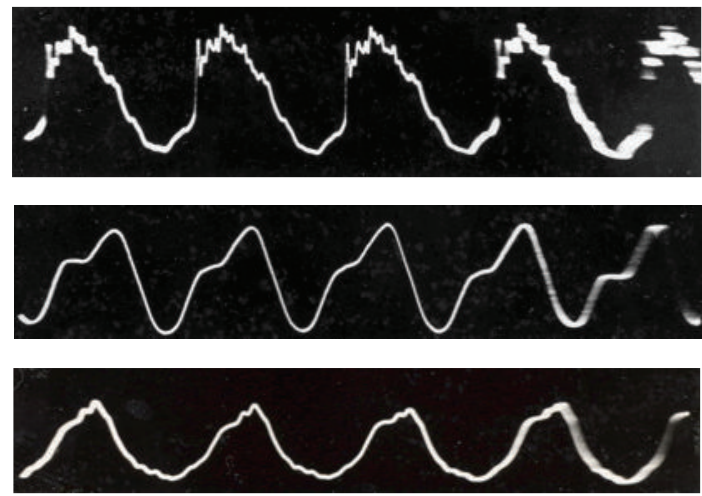

FIGURE 9: Typical pressure oscillograms in steady rotating DW in different points of circle channel (special piezogauges with thermoisolation from hot products [5]).

a radius of a ring charge. Moreover, even for rectilinear free gaseous charge the stationary propagation of multifront DW is possible only in that case, when the diameter of a charge exceeds the "critical" diameter $d>d_{*}$ (or the thickness of a gas charge as a flat mixture layer exceeds the critical magnitude $h>h_{*}$ ). The results of experimental investigations of excitation and quasi-stationary propagation of multifront DW around a cylindrical surface in regime of "exterior" detonation burning were published in [14] at a variation of a radius of a bended surface, thickness of a gas layer, and initial pressure of a mixture (variation of cell size of detonation front).

At initial pressure of a mixture $P_{0}$ be smaller the critical value $P^{*}$ (for the chosen radius of a bended surface as interior radius of a gas charge), it is possible to observe in photos the DW failure and transformation to combustion mode, propagated along circle layer. At $P_{0} \geq P^{*}$ the regimes of DW-reinitiation in points A (marked by arrows on Figure 11) on some distance from the bended boundary are observed. The amount of reinitiation centers is increased with growth $P_{0}$, and they come nearer to a bended surface. Above the value $P^{* *}$ (depending on a curvature radius, on mixture composition, on depth of a gap, etc.) DW "rotation" along circle layer happens in a stationary mode.

The photo on Figure 11 illustrates a possibility of a realization of "exterior" burning around a cylindrical surface in a detonation mode. Moreover, photo obviously demonstrates the basic mechanism of a wave turn and quasi-stationary propagation of multifront DW in a ring gas charge: for this purpose it is necessary that new reinitiation centers arise periodically in the weakened wave on some distance from a bended surface. In gas charge with the free boundary the propagation of multifront DW is possible, if the characteristic size of a gas layer around a bended surface is sufficient for constant renewing of the microcenters of reinitiation. In [14], the evaluation of values of a critical radius of a curving and 


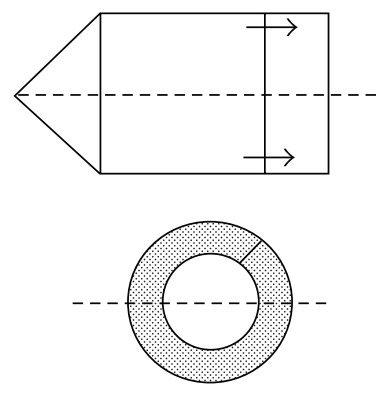

(a)

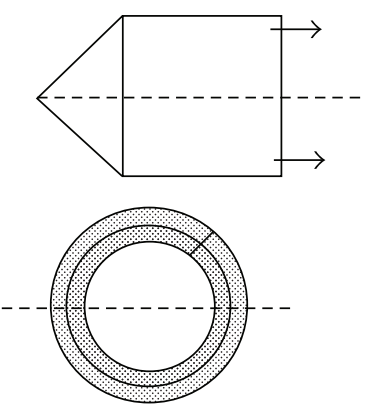

(b)

FIGURE 10: The schema of interchamber burning of a mixture in rotating DW ((a) bounded flow) and schema of "exterior" burning in a ring gas layer with the help of rotating DW ((b) unbounded flow, gaseous charge jet is created at axial or radial injection of a mixture).

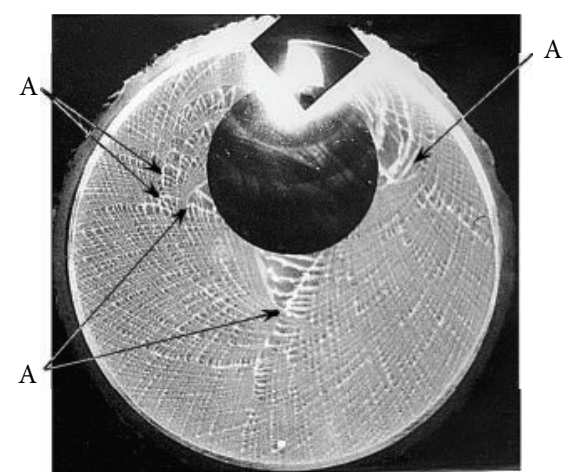

FIGURE 11: A photo of DW "rotation” around of a cylindrical surface. A: Centers of DW reinitiation.

minimum breadth of a ring charge, necessary for a realization of "exterior" burning in detonation mode, was offered: a minimum radius of the interior boundary is $\left(R_{0}\right)_{\min } \approx 0.4 l$, thus a necessary breadth of a gas layer is $\Delta R>0.9 l$. These relations for $\left(R_{0}\right)_{\min }$ and $\Delta R$ can be recommended for practical estimations.

Many important problems for PDE (multicyclical DW initiation in an individual tube of multibarrelled system, the high-frequency regimes, full identity of processes in each cycle, etc.) became less important in the concept of RDE. For example, probable frequency of the PDE schema is limited by length of an individual tube and can be enlarged at the expense of multibarrelled system. At the same time, the frequency in RDE schema is determined by a circle length of a tube only (or diameter). Moreover, in RDE schema the mixture is initiated only in the process beginning, and then it is burned continuously by steady rotated spin DWs. And the self-organizing (autotuning) of a system of spin waves ("multiheaded spin") at a wide variation of flow parameters is an essential virtue of the RDE schema, because it allows to inspect and to operate effectively the DW in RDE. At the same time, the self-organizing of a detonation regime is not inherent in the PDE schema. Consequently, the constant rigid monitoring on engine parameters should be carried out at variation of mixture parameters, that it has not left the operating regime.

\section{Initiation: Ignition, Deflagration to Detonation Transition, Detonation}

The theoretically justified advantages of a detonation burning could be realized in PDE on active fuel-oxygen mixtures (FOM), as FOM are characterized by rather small magnitudes of initiation energies (under condition of ideal intermixing of a mixture at separate injection of components). However, the major condition of practical application of any engine (including those operated on detonation) is the use of an atmospheric air and work on fuel-air mixtures (FAM). For example, using hydrogen as most perspective fuel (from power and ecological points of view) for ignition of stoichiometric FAM at normal conditions $0.017 \mathrm{~mJ}$ is required approximately, and for direct initiation of a detonation regime about $4000 \mathrm{~J}$ is required accordingly (about one gram of TNT) [15]. It is obvious that it is unreal from a practical point of view to realize similar initiation of a detonation for highfrequency mode of operations of multibarrelled schema of PDE.

According to a modern classification the excitation of a combustible mixture is achieved by three basic modes:

(i) the weak initiation (ignition), when only laminar burning is raised with velocities of propagation at a level of tens centimeter in second;

(ii) the strong (direct) initiation, when self-sustained DW is formed in immediate proximity from the initiator and then is spreaded on a mixture with a velocity at a level of several kilometers in second;

(iii) an intermediate case, when the mixture only is ignited on the initial stage and then the front of a flame is accelerated by virtue of the natural or artificial reasons up to visible velocities at a level of hundred meters in second. Under certain conditions further, the deflagration to detonation transition (DDT) can be realized even.

The effect of excitation of combustion or detonation usually carries a "threshold" character ("yes" or "not") for any initiator (Figure 12). The minimum energy ensuring 100 -percentage excitation at the given condition traditionally refers to the critical energy. The type of symmetry is very 


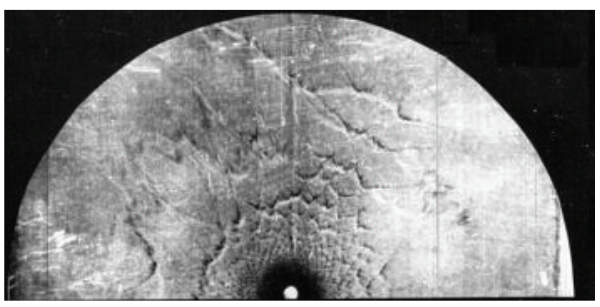

(a)

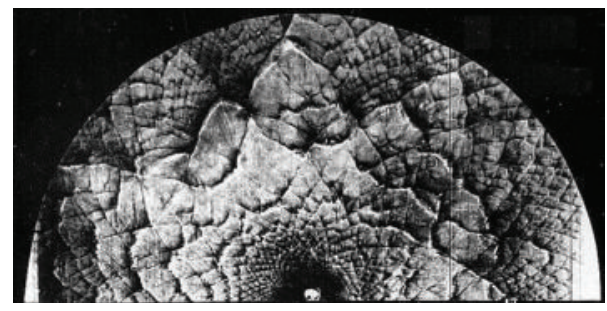

(b)

FIGURE 12: Smoked-foil imprints illustrated the threshold character of initiation of cylindrical DW: (a) DW destroyed at $E<E^{*}$ (subcritical regime), (b) successful DW initiation at $E \geq E^{*}$.

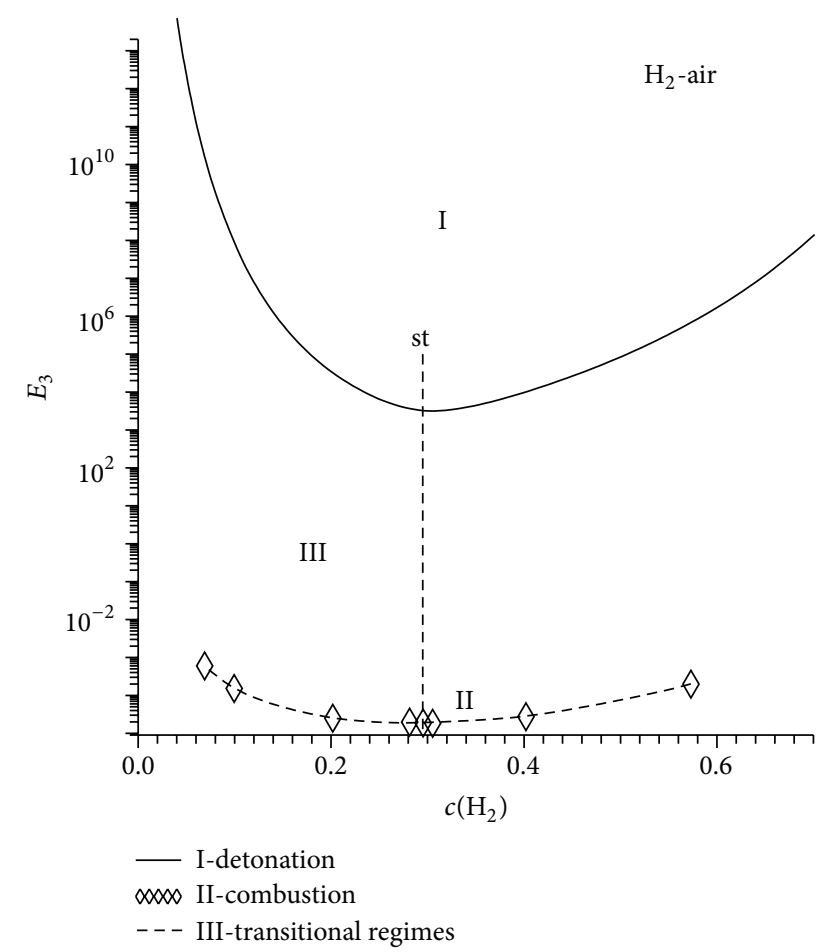

FIGURE 13: The graph of critical energies of ignition and initiation of detonation on molar fuel concentration for hydrogen-air mixtures.

important for initiation: expanding waves (cylindrical or spherical) or quasi-plane (propagation in a rectilinear tube). In expanding waves of laminar burning without artificial action on a wave the basic mechanism of acceleration is autoturbulization of a flame front. The question on a possibility of self-dependent DDT in expanding waves until now is debatable and has not precise experimental confirmation. In pipes alongside with autoturbulization of a flame front the processes of interaction with side walls play rather important role and the principal possibility of DDT is well known (especially for active FOM). Despite an establishment of the DDT fact the data about DDT in pipes are rather inconsistent, especially for FAM.

Usually the critical energy is characterized by some curve which has been carried out on boundary values of energies in area "yes" or "not" ("go" or "no go") on the graph of an initiation energy in dependences on any parameters of

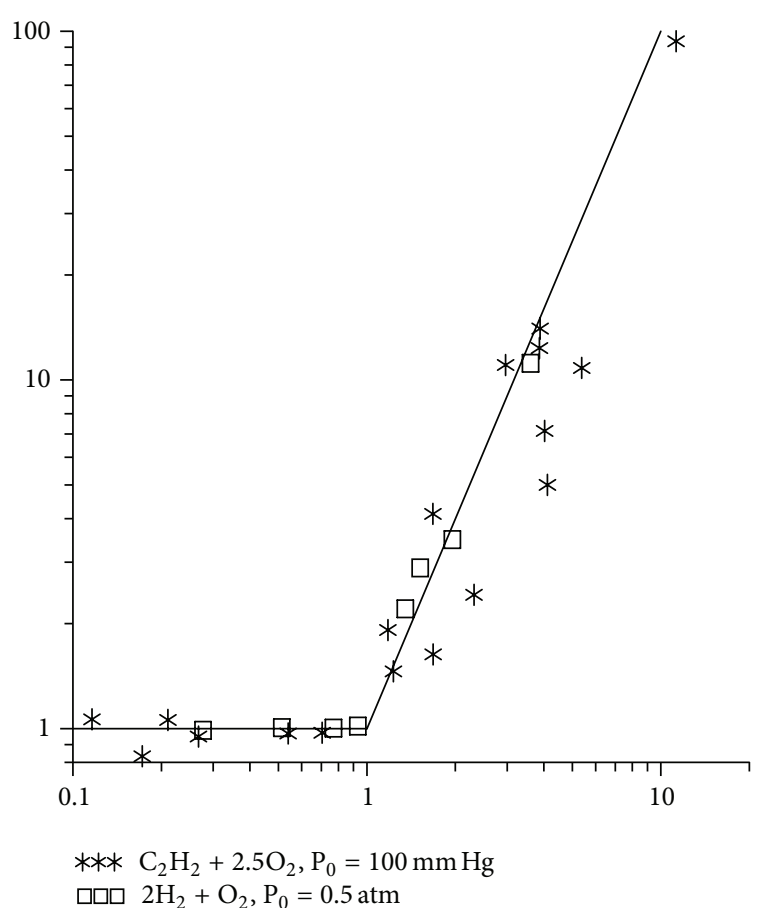

FIGURE 14: Critical initiation energy ( $y$-axis) on duration of discharge ( $x$-axis), both normalized on values for ideal initiator.

a mixture (initial pressure or temperature, duration of a discharge, inter-electrode distance, etc.). For example, on Figure 13 the critical excitation energy of a spherical flames (dashed curve II and above area) and direct initiation of a spherical detonation (solid line I and area above it) for mixtures of hydrogen-air dependent on molar concentration of hydrogen $\left(\left[E_{3}\right]-J\right)$ are presented. The vertical dashed straight line corresponds to stoichiometric ratio of hydrogen with air. The area III between lines I and II, corresponds to excitation of regimes, intermediate between low-velocity laminar flame and high-speed self-sustaining detonation. Between lines I and II the boundary line, corresponded to the DDT processes, should be located.

The critical ignition energy $E_{\text {flame }}$ (anyway, at spark ignition) traditionally appears as a basic parameter of fire hazards of a mixture (first case of the previously-mentioned classification). The critical energy of initiation of a detonation 


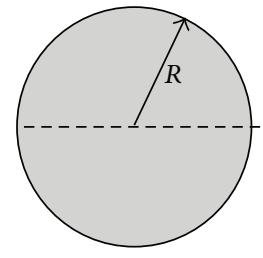

(a)

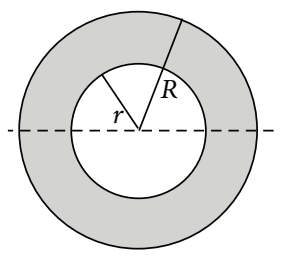

(b)

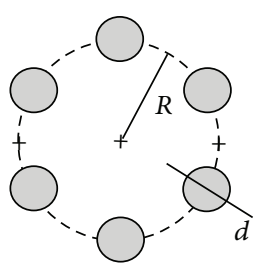

(c)

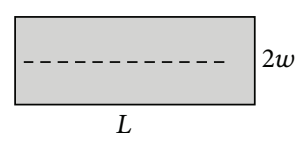

(d)

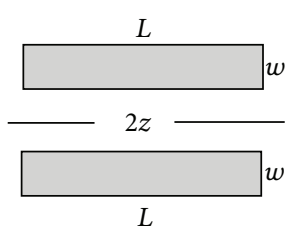

(e)

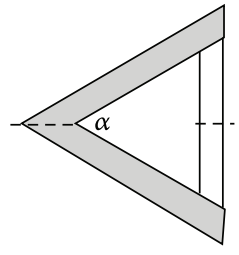

(f)

FIGURE 15: Simplest schema of space distribution of input energy.

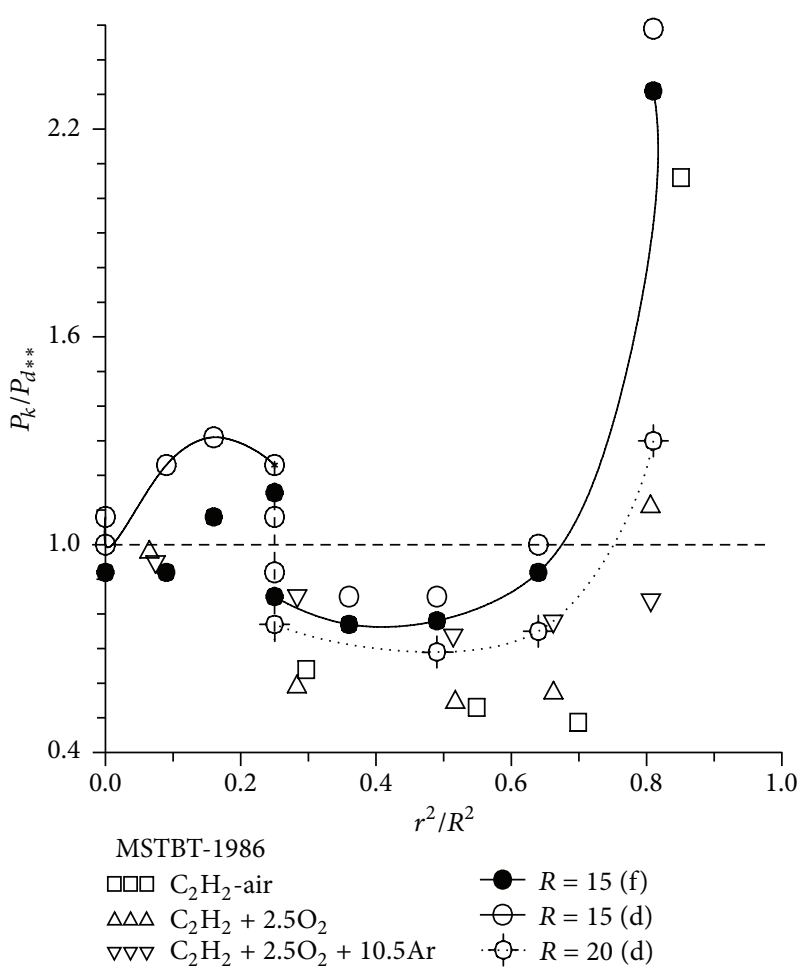

FIGURE 16: Initial pressure of mixture for critical initiation of quasispherical DW by annular charge Figure 15(b) (normalized on pressure for ring charge of the same diameter Figure 15(a)) on blockage ratio (relation among inner and external radii of annular charge).

$E_{*}$ of ideal initiator (from point of view of spatial-temporary characteristics of the initiator) serves as a measure of detonation danger of mixtures: the $E_{*}$ smaller, the mixture more dangerous.

The ideality of the initiator is understood in the following sense. Each mixture at given conditions (pressure, temperature, composition,...) are characterized by some characteristic spatial and temporal scales $r_{*}$ and $t_{*}$ (e.g., induction size and duration). At the same time, at given conditions, the explosive mixture absorbs some energy $E_{v}$ from the initiator (during a finite time interval $t_{0}$ in finite area of space $V_{0}=f(r)$ ), part $\eta$ of an energy $E_{0}$, initially accumulated in the initiator:

$$
E_{v}=\int_{0}^{t_{0}} \int_{0}^{V_{0}} \varepsilon(t, V) d t d V=\eta E_{0},
$$

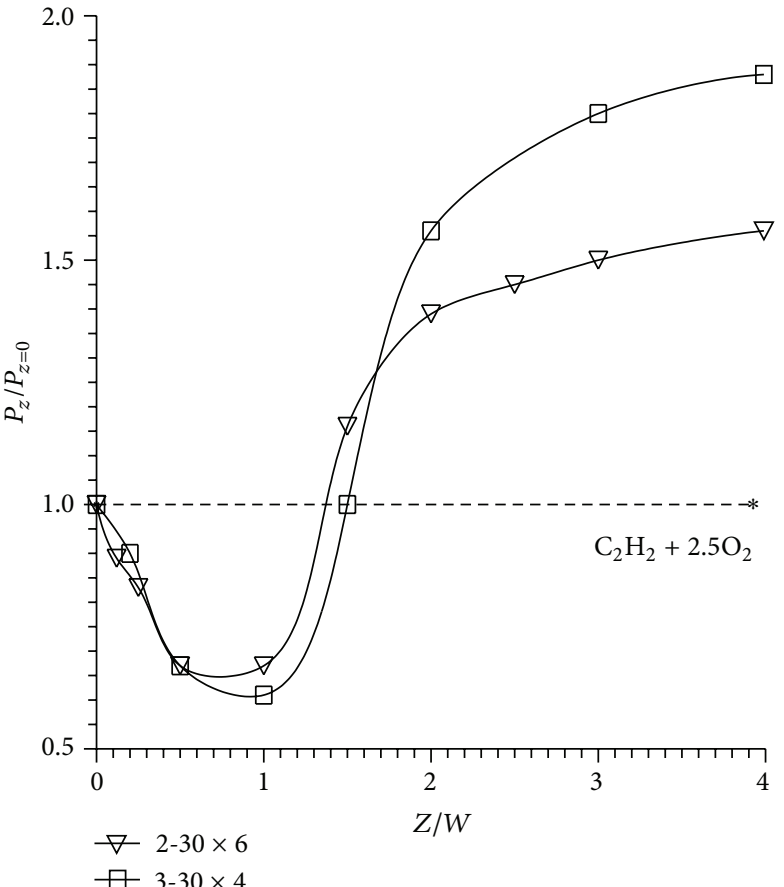

FIGURE 17: Initial pressure of mixture for critical initiation of quasi-cylindrical DW by two or three parallel charges Figure 15(f) (normalized on pressure for the summary charge Figure 15(d)) on distance among charges (normalized on character width of individual charge).

where $\varepsilon(r, t)$ is a function, circumscribing the spatialtemporary law of an entered energy, $v$ is index of dimensionality $(\nu=1,2,3$ for plane, cylindrical and spherical symmetries accordingly). Generally $E_{\gamma}$ is a composite function from characteristic scales of a mixture and initiator (more exactly from their relations). And only under conditions $t_{0} \leq t_{*}$ and $r_{0} \leq r_{*}$, the excitation of multifront DW will be determined by $E_{*}$ only (see later). These conditions represent the criterions of an "instantaneity" and "punctual" of the used initiator and given mixture.

It is necessary to notice that with formal mathematical point of view the previously-stated integrated equality represents a typical functional in a variational task about minimization of an energy $E_{v}$. At mutual influence of the spatial and temporal factor the requirement of minimization of an entered energy up to magnitude $E_{\text {min }}$ is reduced 


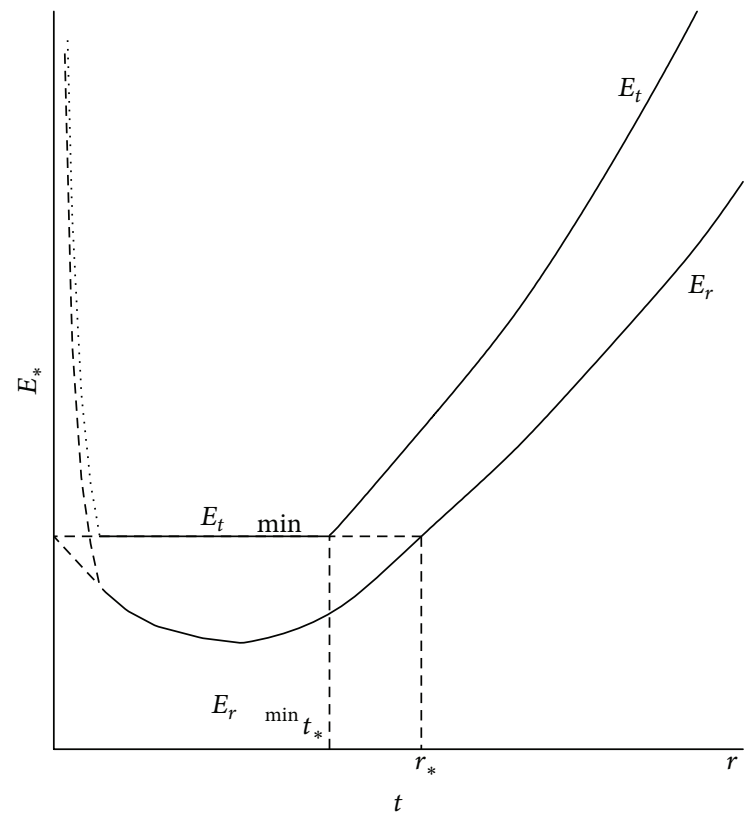

FIGURE 18: Spatial and time components of critical initiation energy on spatial-time parameters of initiator.

simultaneously to minimization of a power density up to $\varepsilon_{\min }=\varepsilon\left(r^{*}, t^{*}\right)$. At the account only of temporal factor $\left(E_{t}\right)$ simultaneously with $E_{\min }$ it is required to optimize a power of input energy $\varepsilon_{\min }=\varepsilon\left(t^{*}\right)$. The influence of a spatial component of input energy $\left(E_{r}\right)$ should be reduced to optimization of a density of an entered energy $\varepsilon_{\min }=\varepsilon\left(r^{*}\right)$ simultaneously with $E_{\text {min }}$.

The knowledge of spatial-temporary characteristics of energy release of any individual initiator is necessary for the correct experimental definition of a critical energy and its optimization.

\section{Optimization of an Initiation: Role of the Spatial and Temporal Factors}

The problem of initiation is extremely important not only for PDE, but also as the interdisciplinary task from a point of view of scientific, practical and ecological aspects of safety. The main aim is the determination of the critical initiation energy and optimization of initiation from a point of view of spatial distribution of an energy, entered into a mixture, and temporal performances.

The following gas-dynamic and kinetic parameters of an initiation task are determinanted:

medium: $r, t, P_{0}, \rho_{0}, \gamma_{0}$ (coordinate, time, pressure, density, ratio of specific heats);

initiator: $t_{0}, r_{0}, E_{v}$ (temporal and spatial parameters of an energy, entered into a mixture, energy, type of a symmetry);

combustible mixture: $t_{\text {ind }}, t_{\text {react }}, E_{\text {act }}, Q, \ldots$ (induction and reaction times, activation energy, specific energy release (chemical)).
Traditionally used simplifications and obtained conclusions are the next:

(1) inert medium: $\left.t_{\text {ind }}=0, t_{\text {react }}=0, E_{\text {act }}=0, Q=0, \ldots\right)$. Then at additional conditions of $P_{0}=0, t_{0}=0, r_{0}=$ 0 the self-similar analytical solution is obtained $r=$ $f\left(t, E_{v}, \rho_{0}, \gamma_{0}\right)$. At $t_{0}>0, r_{0}>0, P_{0}>0$ : the nonself-similar solution is obtained (and the numerical procedures);

(2) combustible mixture: at $t_{\text {ind }}=0, t_{\text {react }}=0, E_{\text {act }}=$ $0, Q>0$ we have the model of a wave with an instant chemical reaction at the front and the selfsimilar analytical solution as steady propagated flame or detonation, even at a zero excitation energy; if $t_{\text {ind }}=0, t_{\text {react }}>0, E_{\text {act }}>0, Q>0, \ldots$, non-selfsimilar solution is obtained.

The variational task about an energy $E_{\gamma}$ of the abovementioned integrated relation can experimentally be investigated by several paths.

(1) If there is a variation of duration of energy input at conservation of spatial sizes of area of energy input: $V_{0}=$ const, $t_{0}=$ var, then a power density is transformed to a power: $\varepsilon(t, V) \Rightarrow \varepsilon(t)$. Nondimensional critical energies of initiation of a cylindrical detonation independent from dimensionless duration of an electrodischarge (the data [16]) are submitted on Figure 14 for stoichiometric mixtures of hydrogen and acetylene with oxygen. The critical initiation energy is a unique criteria parameter of a mixture only for a case, when the duration of an initiating discharge does not exceed some critical magnitude; otherwise, the more large energy is required for DW initiation, then the discharge is more long. In other words, at $t_{0}<t^{0}$ $\left(t^{0}:\right.$ the characteristic temporal scale of a mixture) it can be spoken about "quasi-ideal" initiator: only in this case; the initiation is defined by a unique parameter, the critical energy $E_{v}^{*}$ !. At $t_{0}>t^{0}$ the initiator should be referred to as imperfect. At anyone $t_{0}$ the critical initiation carries a threshold character: damping at $E<E^{*}$ and DW excitation at $E>E^{*}$.

(2) Conservation of duration of energy input and both variations of a size and form of area of energy input: $t_{0}=$ const, $V=$ var, $\varepsilon(t, V) \Rightarrow \varepsilon(V)$, power density is transformed to an energy density.

The simple forms of the initiators at investigations of influence of spatial distribution of an entered energy for the critical initiation energy of spherical DW are submitted on Figure 15. Initiator charges can be modeled in the following geometric forms: (a) a circular disk with radius $R$; (b) an annulus with radii $r$ and $R$; (c) a multipoint scheme represented by $n$ charges of diameter $d$ spatially distributed, for example, on a circle of radius $R$; (d) a single rectangular plane charge with $L \cdot 2 w$ sizes; (e) a system of parallel line charges of $L \cdot w$ sizes, separated by a distance $2 z$; or (f) a system of nonparallel line charges, for example, shaped with an angle $\alpha$ or closed triangle. 


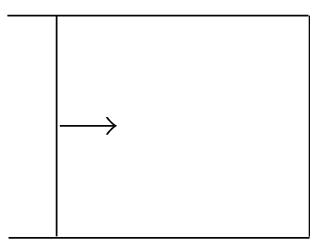

(a)

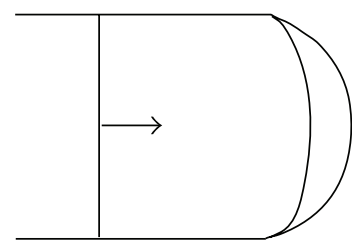

(b)

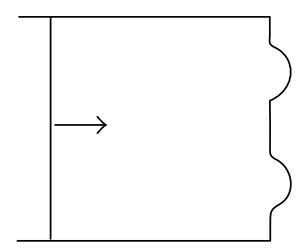

(c)

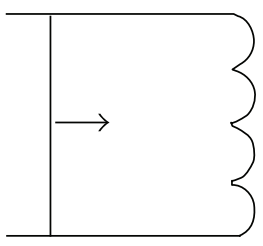

(d)

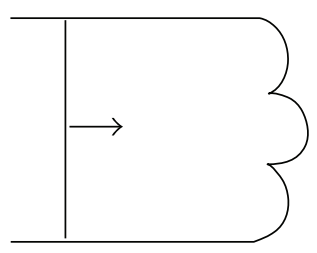

(e)

FIGURE 19: The typical forms of concave surfaces for optimization of initiation at SW reflection: (a) plane reflector; (b) single ring or elliptical reflector on whole cross-section or single ring or elliptical reflector on limited part of cross-section; (c) two ring or elliptical reflectors shifted one another; (d) some neighboring elliptical reflectors located along plane; (e) some ring or elliptical reflectors located along concave elliptical line (neighboring or with shift).

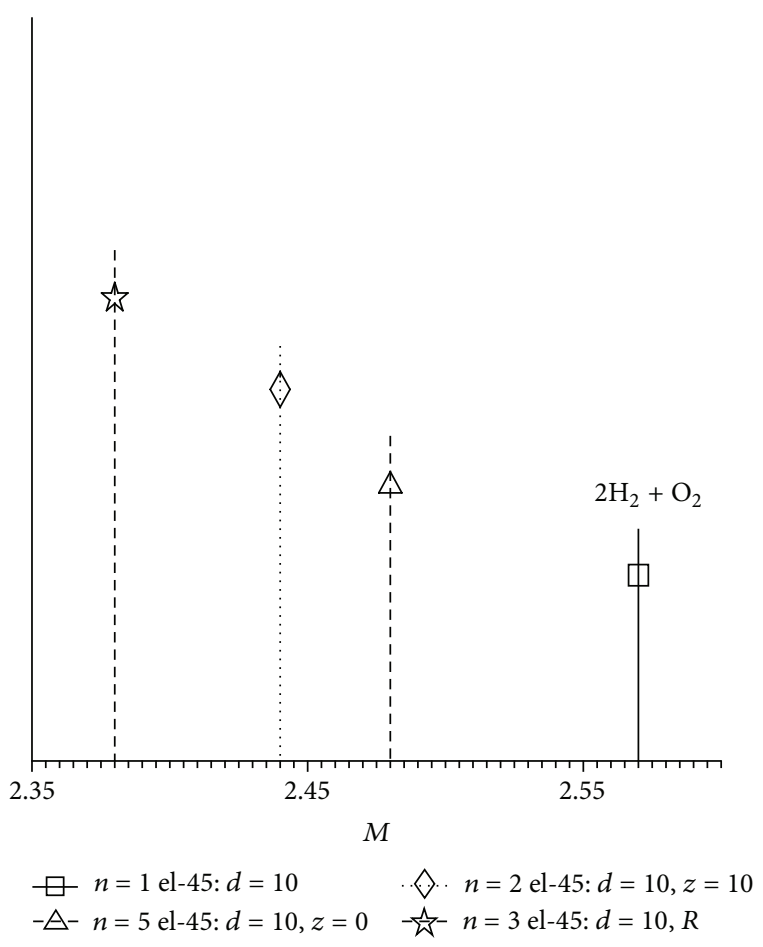

FIGURE 20: The critical (lowest) Mach number for excitation of a detonation at interaction of an incident SW with various reflectors: identical mixture conditions and variation of SW Mach number for individual reflector. The area from the right of vertical line corresponds to successful initiation ("go", "yes"), from the left to unsuccessful initiation ("no go").

The critical conditions of initiation of a spherical detonation by ring charges are submitted on Figure 16, $r$ and $R$ : interior and exterior radii of a charge. The area of a below dashed horizontal line corresponds to the greater effectiveness of ring charges in comparison with a compact charge in form of disk of radius $R$. The similar effect of the greater effectiveness of the distributed charges in a comparison with a compact charge is observed and at initiation of cylindrical DW by two or three parallel linear charges-Figure 17 (zdistance between charges).

On Figure 18 the influence of a temporal component to an initiation energy is submitted: at variation of duration of initiating impulse, the value $E_{t}=$ const at $t_{0}<t^{0}$,

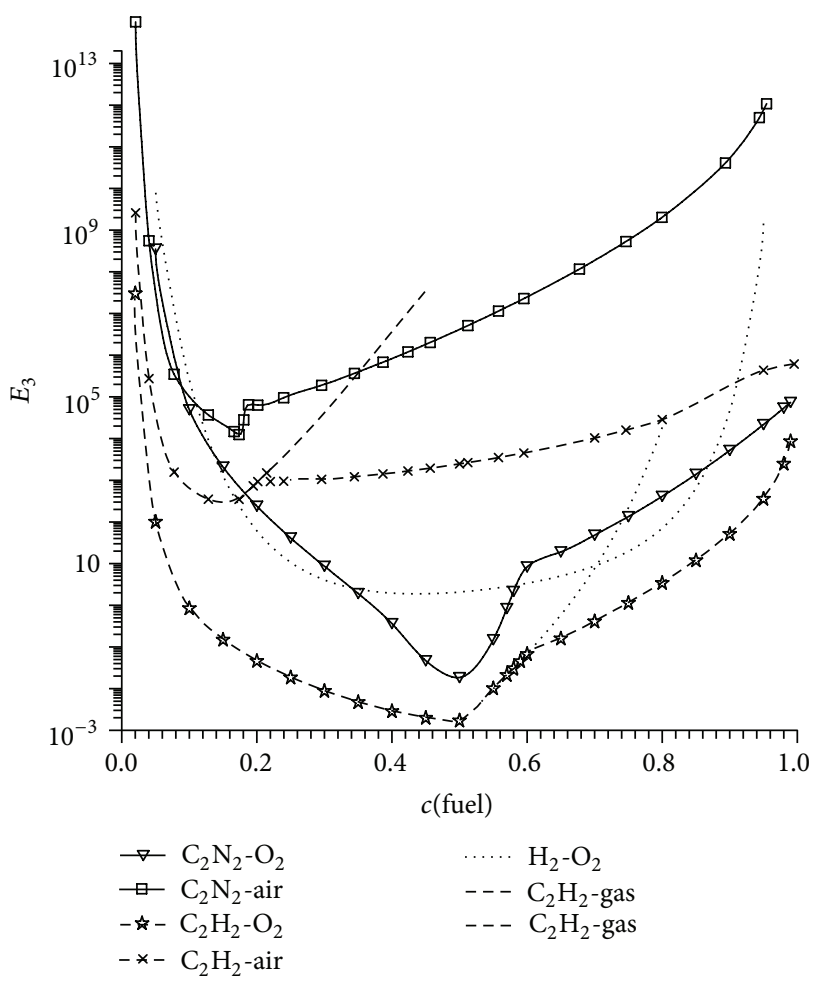

FIgURE 21: The graphical BANK of data about critical initiation energy of spherical DW (J) on fuel molar concentration for different mixtures, which allow to compare mixtures on its detonation hazards. Doubled lines for $\mathrm{C}_{2} \mathrm{H}_{2}$ correspond to cases of gaseous and solid $\mathrm{C}$ atoms in detonation products.

at $t_{0}>t^{0} E_{t}$ will increase. Spatial component $E_{r}$ is characterized by the U-figurative form with optimum magnitude; that is, the critical initiation energy can be reduced at the expense of spatial distribution. For last case, the problem on a choice of the characteristic scale of a mixture $r^{0}$ is very important.

The optimization of initiation can be reached with the help of many methods, for example;

(a) spatial initiation, including the multicharge schema with a variation of an amount of charges and their spatial disposition from each other; 
(b) initiation by a series of impulses with a variation of amplitude and duration of individual impulse and also their off-duty factor;

(c) initiation at reflection of shock wave (SW) from a focusing surface;

(d) initiation by jets of hot and active substances, including ionized components;

(e) application of promoters;

(f) using mixtures with gradients of parameters (density, temperature, and composition).

For example, in Figure 19, the initiation with the help of $\mathrm{SW}$ reflections from concave surface is illustrated. Figures 19(a) and 19(b) are classical schema of SW reflection from a plane surface and from cylindrical (or elliptic-dashed profile) surface (e.g., $[17,18])$; the symbols (in order) correspond to one elliptic reflector with an effective diameter of $10 \mathrm{~mm}$; to five adjoining elliptic reflectors with a similar size (as on Figure 19(d)); to two elliptic reflectors of an identical effective diameter that moved from each other on $10 \mathrm{~mm}$ (the schema Figure 19(c)); and to three elliptic reflectors located along a concave elliptic curve, "double" focusing of SW (as on Figure 19(e)). The additional positive effect in decreasing the critical Mach number of the incident SW can be reached with the help of multifocused system (MFS), when a few concave reflectors are located on the round or elliptic surface (Figures 19(c)-19(e)). In this case the focusing of the reflected waves creates new "hot spots" as the micro-initiators, which initiate mixture immediately after SW focusing, or later on the following stage of interaction by secondary SWs, generated by "hot spots." Centers of individual reflectors can place as along a direct line (Figure 19(d)), as and along concave curve (Figure 19(e)), and also with some shift $z$ from each other (Figure 19(c)). Three last schemas are more effective at DW initiation comparison with cases Figures 19(a)-19(b). In Figure 20, the experimental data about limiting Mach number of an incident shock wave are presented, at which the initiation of a mixture is observed: decrease of the critical Mach number of the incident SW for DW initiation is precisely fixed.

\section{BANK of Detonation Danger}

The critical energy (as a unique parameter describing excitation of a mixture by an ideal initiator) attracts an attention of many investigators until now. Three types of models are known for estimations of the critical initiation energy:

(1) model of one-dimensional initiation for numerical accounts as a system of the one-dimensional equations of a gas dynamics and kinetics;

(2) semi-empirical models of initiation of one-dimensional DW (about 20 variants);

(3) model of initiation of multifront DW.

The one-dimensional numerical calculations were carried out for the limited amount of mixtures (about ten), while the practice interest requires the analysis of hundreds of

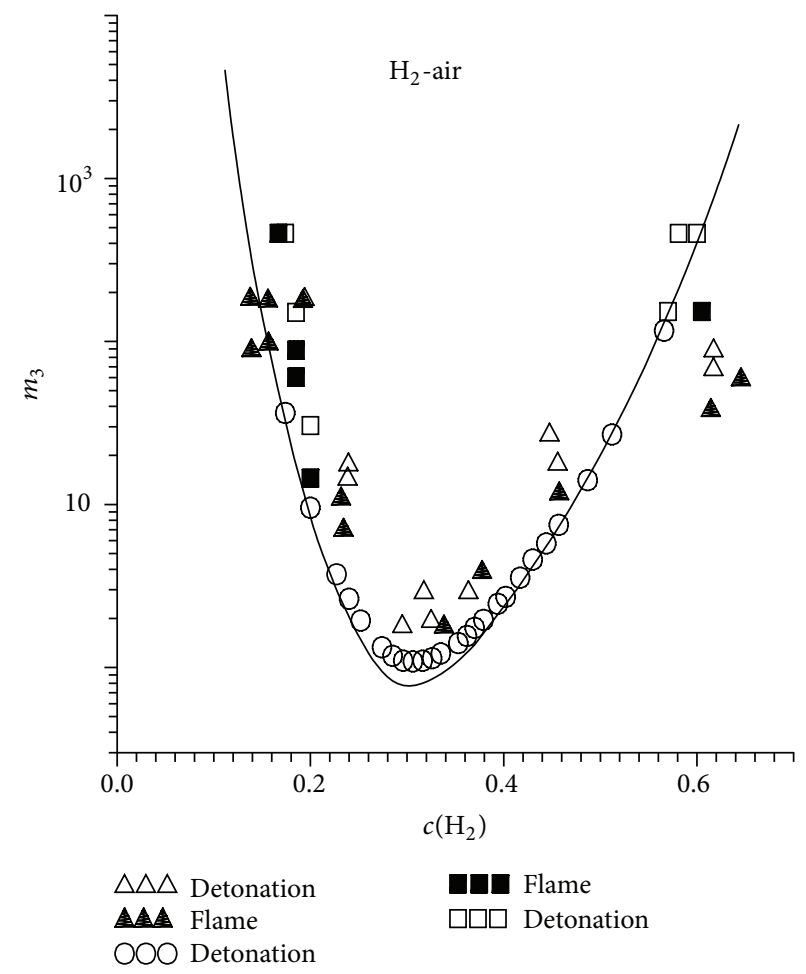

Figure 22: Correlation of experimental and calculated data about critical mass of TNT charge for initiation of spherical DW $\left(\left[m_{3}\right]=\right.$ g) on fuel molar concentration of $\mathrm{H}_{2}$-air mixtures $c\left(\mathrm{H}_{2}\right)$.

various mixtures. In LIH (Novosibirsk, Russia), the code "SAFETY" is developed for the similar purpose, in which all known models of a type (2)-(3) (e.g., $[15,19])$ are included. The calculated data allow to create the BANK of detonation danger of various combustible systems Figure 21. As an example, on previously-mentioned Figure 13 the graphic interpretation of calculated data about critical energies for initiation of a detonation process and mixture ignition for hydrogen-air mixtures is presented. The critical mass of TNT charge (in gram) for initiation of a spherical detonation in hydrogen-air mixtures independent from molar concentration $\mathrm{c}$ of fuel in a mixture is submitted in Figure 22. The good correlation of calculated and experimental values is visible. The detailed analysis of experimental data on different detonation parameters was published in review [20].

\section{DDT Optimization}

The prospects of application of a detonation in various technological processes (as fastest regime of a mixture burning) are stroke on difficulty of its practical realization, because the direct initiation of DW in FAM, as a rule, is achieved with the help of high explosive (HE) charges. The ignition of a mixture by the initiator of low power and consequent artificial acceleration of a flame with the help of highly effective accelerators down to DDT is natural alternative to HE charges.

It is necessary especially to underline that the DDT accelerator should guarantee full identity of DDT process in 


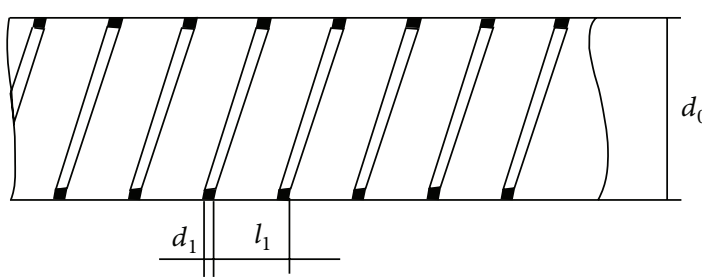

(a)

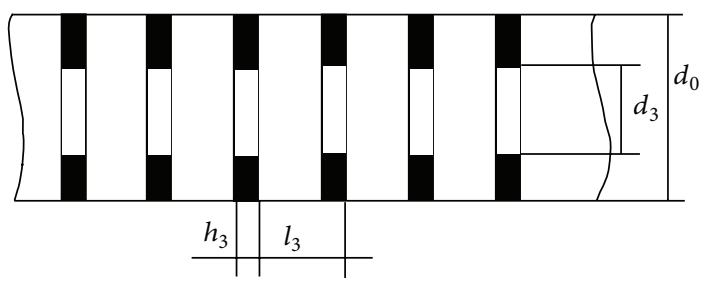

(c)

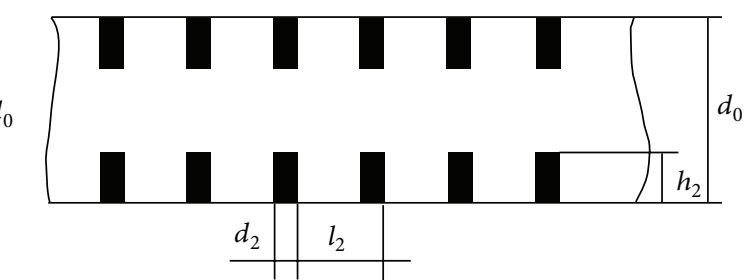

(b)

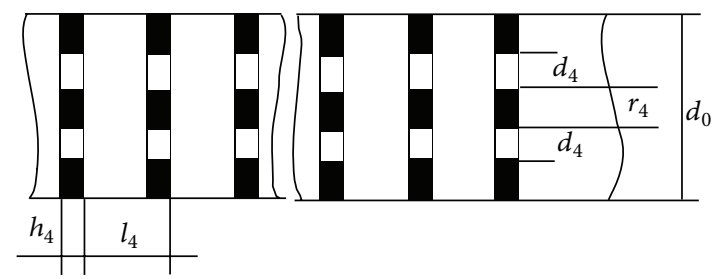

(d)

FIGURE 23: Typical schema of use of passive elements for acceleration of "plane" flames: helixes (Tshelkin spiral), each helix is characterized by three parameters with the dimensions of length: tube diameter $d_{0}$, thickness $d_{1}$, and pitch $l_{1}$ of spiral (a); singly standing rods ("forest" schema) (b) (in addition to the dimensions of an individual rod such as diameter $d_{2}$ and length $h_{2}$, the rod-type accelerator is characterized by the number $n$ of the used rods and a law of their distribution in the tube, for example, by the step along the axis, azimuthal displacement in neighboring planes, etc.); disks with holes coaxial (c) (the characteristic dimensions are thickness $h_{3}$ and displacement $l_{3}$ of disk, and hole diameter $d_{3}$; the number of holes is of importance also), or with displacement (the dimensions are thickness $h_{4}$ and displacement $l_{4}$ of disk, hole diameter $d_{4}$ and distance $r_{4}$ among holes) (d).

each individual tube for each cycle of PDE work. Moreover, the initiation should be effectively realized in a high-speed stream of a mixture (subsonic or supersonic) instead of classical initiation of a motionless mixture. It is not evident that the conditions of DW initiation for a motionless mixture and for chemically active stream are identical. Therefore, the additional experimental researches of initiation of highspeed streams of FAM, for example, with the help of wind tunnel techniques in a wide range of the flow Mach numbers are necessary. The similar investigations on optimization of DDT accelerators for FAM are necessary also.

On a today the limited number of correct experimental measurements of the critical ignition energy is known from the literature (it is rather strange!).

We would like to pay attention once again to a possibility of a decrease on some order of the critical initiation energy at simultaneous optimization of the spatial and temporal factors of an entered energy. But it is necessary to underline especially that such decrease is reached at quite defined relations between spatial and temporal parameters of an initiator and spatial: temporal parameters of a combustible mixture. The high effectiveness is reached at an optimum relation between these values, otherwise special effectiveness is not observed. In the literature, more often, only the Tshelkin spiral is used as a basic element for acceleration of DDT process. But even such spiral is characterized by three parameters with dimensionality of length: by an exterior diameter of a spiral (usually equals diameter of a pipe), thickness of a wire, and pitch of a spiral. And only at optimum relations of these parameters with a spatial parameter of a combustible mixture the action on DDT will be highly effective, and at an arbitrary relation the influence of a spiral on DDT is insignificant.
In LIH during the previous years the effective accelerators of DDT for FOM and also the accelerators for excitation of spinning DW in FAM were developed: after ignition of a mixture by an automobile spark the action of the accelerator leads to formation of the spinning DW on a distance of 16 calibers in equimolar hydrogen-air mixture and in stoichiometric methane-air mixture on distance of 25 calibers.

The turbulence plays an important role in intensify of burning (at expense of an increase of a flame surface). Two basic modes of a flame turbulization are known: natural (autoturbulization) and artificial turbulization with the help of various obstacles. Usually an autoturbulization produces a slow acceleration of flame front, and interactions with obstacles and pipe walls are more effective, especially with concave boundary. Some typical schema of use of passive elements for acceleration of "plane" and divergent flames were presented on Figures 23-24. For forming of multifront DW it is necessary that the elements of the accelerator were distributed uniformly on a cross-section of a detonation pipe. Moreover, the elements must repeat the action on combustion front at its acceleration along a detonation tube over and over again. The given idea was realized in [21] with attraction of the theory of turbulent jets.

At a flow of a streamline body by gas behind a body the turbulent layer is formed with the extending boundaries: exterior from a stream axes and interior on direction to axes (Figure 25). The interior boundaries are closed on axes on some distance from streamline body (points 1 on Figure 25), and the point of intersection is defined by a body size and by expansion angle of the interior boundary. If in the given cross-section the screen ("lattice") from a few body is located, 


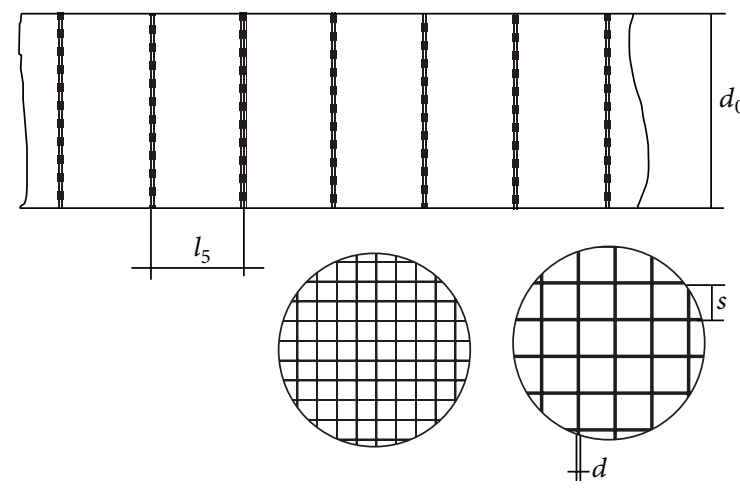

(a)

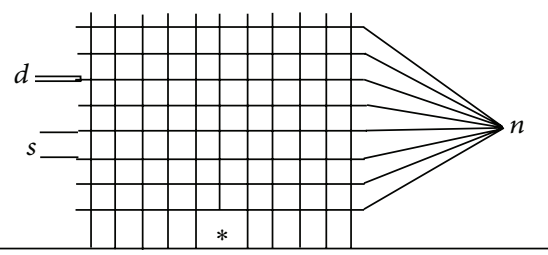

(c)

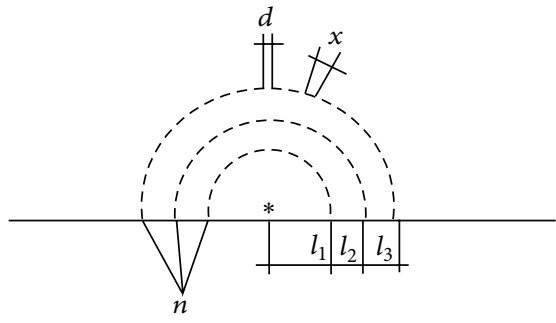

(b)

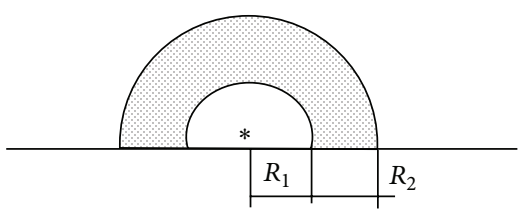

(d)

FIGURE 24: Typical schema of use of passive elements for acceleration of "plane" and divergent flames: wire or rod screens (a) (the characteristic parameters are the diameter of the wire $d$, the permeability $s$ of the screen, and the distance between the screens $l_{5}$ ); hemispherical shells with holes (b) (parameters are hole diameter $d$ and holes displacement $x$, number of hemispheres $n$, and their spatial arrangement $l_{1}, l_{2}, l_{3}, \ldots$ ); $3 \mathrm{D}$ rod-structures (c) (parameters are rod diameter $d$ and permeabilitys, number of rods $n_{i}$ (total and in the planes); obstacles from porous materials (d) (radii $R_{1}$ and $R_{2}$, porosity, number of porous layers,...), and so forth.

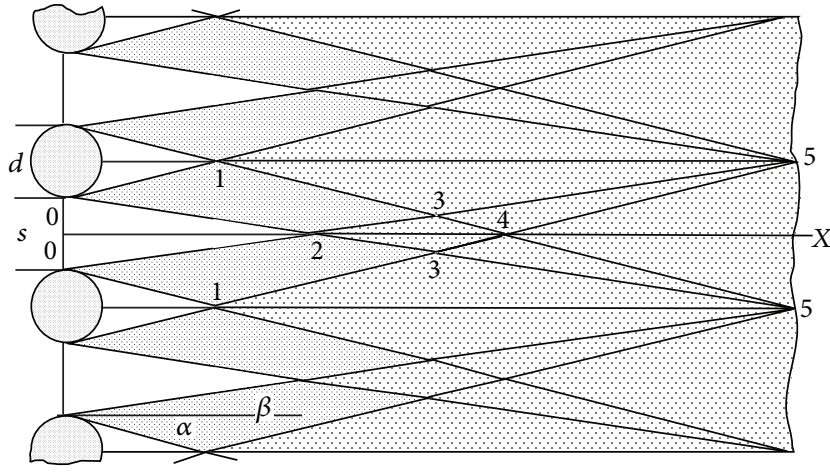

FIGURE 25: Scheme of flow turbulization behind the obstacles.

then along with intersections of the interior boundaries on axes of each body, the exterior boundaries of turbulent layers from the neighboring bodies will be closed also. The intersection point of the exterior boundaries is defined as a distance between bodies and by expansion angle of the exterior boundary (points 2 on Figure 25). The optimization condition was formulated as an equidistantness of intersection points of the interior (1) and exterior (2) boundaries of turbulent layers from a plane of "lattices." This condition allows to establish an optimum relation between geometric parameters of individual bodies (as elements of the accelerator) in the given cross-section and also to determine the coordinates of following "lattice" with turbulization elements. Such relations represent an engineering technique for purposeful projection of turbulent device for DDT accelerators.

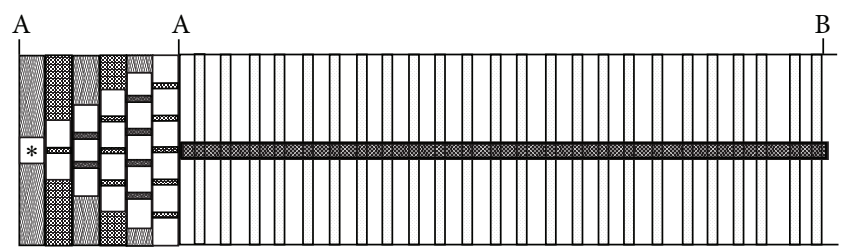

FIGURE 26: Simplest scheme of generator of plane wave and DDT accelerator.

In [21] with the help of similar DDT accelerator (Figure 26) the record of effectiveness was obtained: at pressure $1 \mathrm{~atm}$ for stoichiometric of mixtures of acetylene-air and hydrogen-air the multifront DW in tube of diameter of 250 $\mathrm{mm}$ was formed on a distance of about 2 calibers (Figure 27). The advantage of the highly effective DDT accelerator is also that it allows to transfer the investigations of fuel-air mixtures from target ground conditions (with using of high explosive) to laboratory.

It would mark especially that the spatial distribution of ignition centers allows under certain conditions considerably (on orders) to reduce the DDT distance.

\section{Difficulties in a Realization of a Detonation Engine}

(1) "Weapon" scheme of PDE: problem of direct DW initiation ДВ in fuel-air mixtures: it is necessary especially to underline that the characteristic times of 


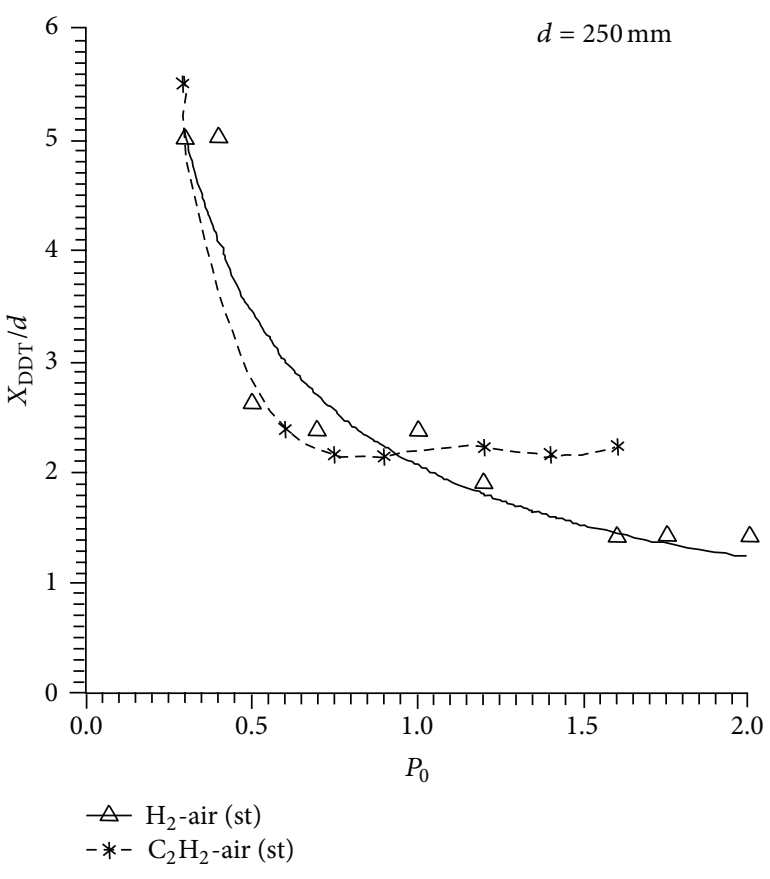

FIGURE 27: DDT distance (normalized on tube diameter) on initial mixture pressure (atm) for some fuel-air mixtures.

DW forming at direct initiation of DW lay in microsecond range, and times of ignition in milliseconds. The times, for which the flame will be transferred in detonation, will make one hundred microseconds at the best. And if, for immovable mixture, it does not play the special role, it becomes defining for flow conditions.

(2) PDRJE: problem of cyclical operation: though the work of such engine on FAM is confirmed experimentally in single cycle, there are doubts in its stability at change of direction of DW propagation and in DW conservation at such change in case of cyclical operation. If DW is broken on some cycle (e.g., at run up to nozzle entering) and is turned to combustion wave, it will be affected on work of an engine and will require immediate operations on restoration of detonation process, for example, with the help of additional system of DW initiation (such system must be in condition of "constant readiness"). It can complicate the control process on an engine.

(3) The scheme of inchamber mixture burning by steady rotating DW removes a set of problems intrinsic to PDE, but the problem on realization of a classical detonation regime remains. During latest years there are some attempts to name any regimes as detonation, if the velocities of which exceed a sound velocity of an initial mixture (in literature, devoted to detonation engine). Such attempts reduce to terminological dissonance. Point is that if the regime has a smaller velocity on a comparison with an ideal C-J velocity (regime of quasi-detonation), the point of contact of the Mikhelson line with an adiabatic curve of energy release at decreasing of DW velocity will be displaced in region of smaller $P-V$, and so pressure in such wave will be lower than pressure in ideal DW. Naturally, it will reduce the integrated work at expansion of products of such "detonation" and the question on the effectiveness degree of such regime requires additional researches and arguments.

(4) Exterior burning: the realization on FOM requires confirmation of serviceability on FAM. Common note to the analysis of effectiveness of various engines and its characteristic cycles, the clearness in definition of process of product expansion (with the frozen or equilibrium state), is necessary: it can essentially influence the obtained values.

The problem of Pulse Detonation Engine and Rotated Detonation Engine is widely investigated in the latest years in many countries. In [22-25], for example, one can read the opinion of the most known specialists of PDE and RDE problems about some specific aspects and perspectives of future investigations of PDE and RDE.

\section{Conclusion}

The basic problems of use of a detonation in propulsion systems with impulse and continuous burning of a combustible mixture are discussed. The basic results on propagation of detonation waves in supersonic streams are presented with references to PFRJE. The results of researches showing a principal possibility of creation of an engine with exterior detonation burning are presented. The base results (concerning PDE) on optimization of initiation are explained at the expense of spatial and temporal redistribution of an energy, entered into a mixture. The technique for projection of highly effective accelerators of DDT is discussed.

\section{References}

[1] K. I. Tshelkin and Ya. K. Troshin, Gas-Dynamic of Combustion, in Russian, USSR Academy of Sciences, Moscow, Russia, 1963.

[2] Yu. A. Nikolaev, A. A. Vasil'ev, and V. Yu. Ulianitsky, "Gaseous detonation and its application in technique and technologies," Combustion, Explosion, and Shock Waves, vol. 39, no. 4, pp. 2254, 2003.

[3] G. D. Roy, S. M. Frolov, A. A. Borisov, and D. W. Netzer, "Pulse detonation propulsion: challenges, current status, and future perspective," Progress in Energy and Combustion Science, vol. 30, no. 6, pp. 545-672, 2004.

[4] V. G. Aleksandrov, G. K. Vedeshkin, A. N. Kraiko et al., "Supersonic pulsing detonation ram-jet engine (SPDRJE) and method of its functioning" (Russian), The patent of Russian Federation no 2157909, October 2000, priority from 26.05.1999.

[5] A. A. Vasil'ev, Investigation of a stationary detonation in the ring channel [Ph.D. thesis], Novosibirsk State University, 1968, in Russian.

[6] V. G. Alexandrov, A. A. Baskakov, G. K. Vedeshkin et al., "Supersonic pulse detonation ramjet engine: new experimental and theoretical results," in Application of Detonation to Propulsion, 
G. Roy, S. Frolov, and J. Shepherd, Eds., pp. 277-282, Torus Press, Moscow, Russia, 2004.

[7] A. A. Vasil'ev, V. I. Zvegintsev, and D. G. Nalivaichenko, "Detonation waves in supersonic flow of reacting mixture," Combustion, Explosion and Shock Waves, vol. 42, no. 5, pp. 85100, 2006.

[8] B. V. Voitsekhovsky, “A stationary detonation,” Reports of USSR Academy of Sciences, vol. 129, no. 6, pp. 1254-1256, 1959 (Russian).

[9] B. V. Voitsekhovsky, "A spin stationary detonation," Applied Mechanics and Technical Physics, vol. 3, pp. 157-164, 1960 (Russian).

[10] F. A. Bykovskii, A. A. Vasilev, E. F. Vedernikov, and V. V. Mitrofanov, "Detonation burning of a gas mixture in radial ring chambers," Combustion, Explosion, and Shock Waves, vol. 30, no. 4, pp. 111-119, 1994.

[11] S. A. Zhdan and F. A. Bykovsky, "Investigations of continuous spin detonations at Lavrentyev Institute of Hydrodynamics," in Pulse and Continuous Detonation Propulsion, G. Roy and S. Frolov, Eds., pp. 181-202, Torus Press, Moscow, Russia, 2006.

[12] F. A. Bykovskii, S. A. Zhdan, and E. F. Vedernikov, "A continuous spin detonation in fuel-air mixtures," Combustion, Explosion and Shock Waves, vol. 42, no. 4, pp. 107-115, 2006.

[13] A. A. Vasil'ev and D. V. Zak, "Detonation of gas jets," Combustion, Explosion, and Shock Waves, vol. 22, no. 4, pp. 463-468, 1986.

[14] A. A. Vasilev, "Characteristic conditions of propagation of a multi-front detonation along a convex surface," Combustion, Explosion and Shock Waves, vol. 35, no. 5, pp. 86-92, 1999.

[15] A. A. Vasil'ev, Near-Limiting Regimes of Detonation, in Russian, Lavrentyev Institute of Hydrodynamics, Novosibirsk, Russia, 1995.

[16] J. H. S. Lee, "Initiation of gaseous detonation," Annual Review of Physical Chemistry, vol. 28, pp. 75-104, 1977.

[17] H. Gronig and B. Gelfand, Eds., Shock Wave Focusing Phenomena in Combustible Mixtures: Ignition and Transition to Detonation of Reactive Media Under Geometrical Constraints, Shaker, Aachen, Germany, 2000.

[18] O. V. Achasov, S. A. Labuda, O. G. Penyazkov, P. M. Pushkin, and A. I. Tarasov, "Initiation of a detonation at a reflection of a shock wave from a concave surface," Journal of Engineering Physics and Thermophysics, vol. 67, no. 1-2, pp. 66-72, 1994.

[19] A. A. Vasilev, "Estimation of the critical initiation energy for ignition and detonation in gaseous mixtures," in Application of Detonation to Propulsion, G. Roy, S. Frolov, and J. Shepherd, Eds., pp. 49-53, Torus Press, Moscow, Russia, 2004.

[20] A. A. Vasil'ev, "Dynamic parameters of detonation," in Shock Wave Science and Technology Reference Library, F. Zhang, Ed., vol. 6, pp. 213-279, Springer, 2012.

[21] A. A. Vasilev, "Optimization of accelerators of deflagrationto-detonation transition," in Confined Detonations and Pulse Detonation Engines, G. Roy, S. Frolov, R. Santoro, and S. Tsyganov, Eds., pp. 41-48, Torus Press, Moscow, Russia, 2003.

[22] W. H. Heiser and D. T. Pratt, "Thermodynamic cycle analysis of pulse detonation engines," Journal of Propulsion and Power, vol. 18, no. 1, pp. 68-76, 2002.

[23] F. Falempin, "Continuous detonation wave engine," in Advances on Propulsion Technology for High-Speed Aircraft, RTO-ENAVT-150, Paper 8, NATO, 2008.

[24] D. A. Schwer and K. Kailasanath, "Numerical investigation of the physics of rotating detonation engines," Proceedings of the Combustion Institute, vol. 33, no. 2, pp. 2195-2202, 2011.
[25] M. Hishida, T. Fujiwara, and P. Wolanski, "Fundamentals of rotating detonations," Shock Waves, vol. 19, no. 1, pp. 1-10, 2009. 

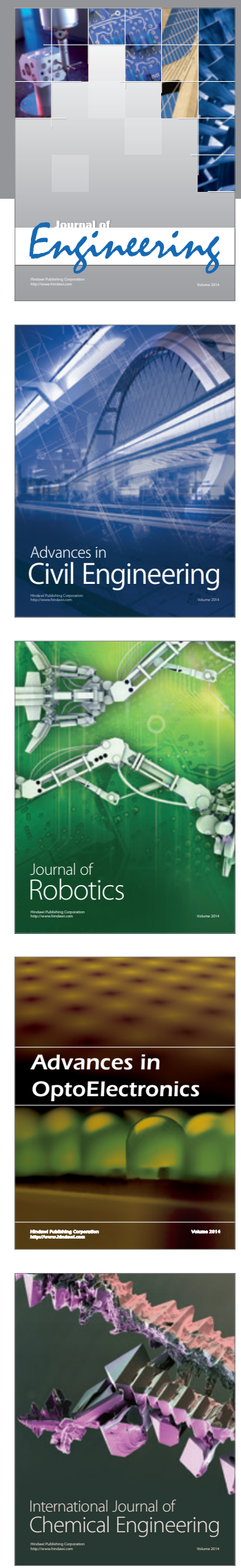

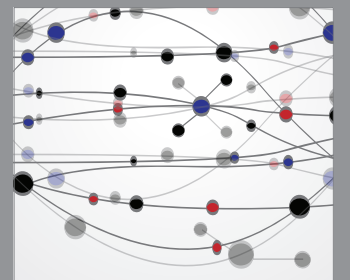

The Scientific World Journal
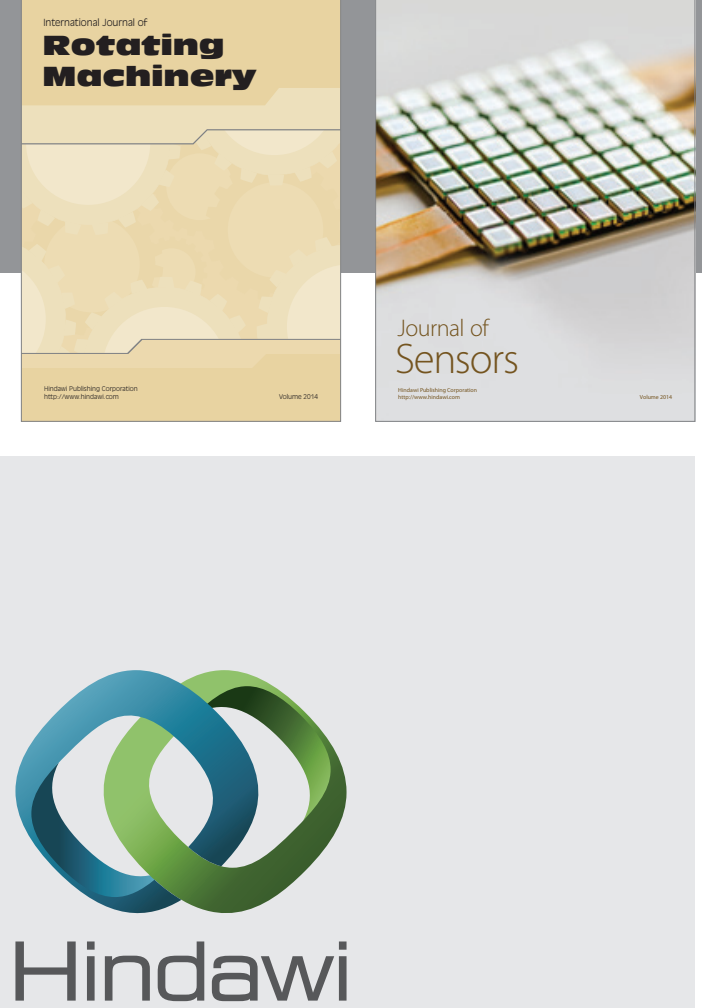

Submit your manuscripts at http://www.hindawi.com
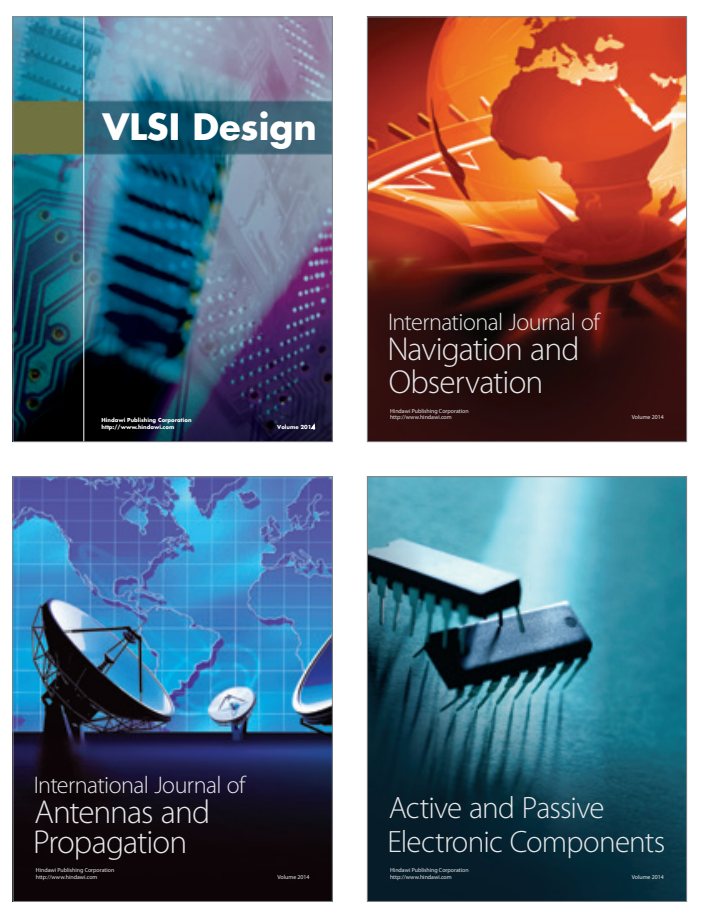
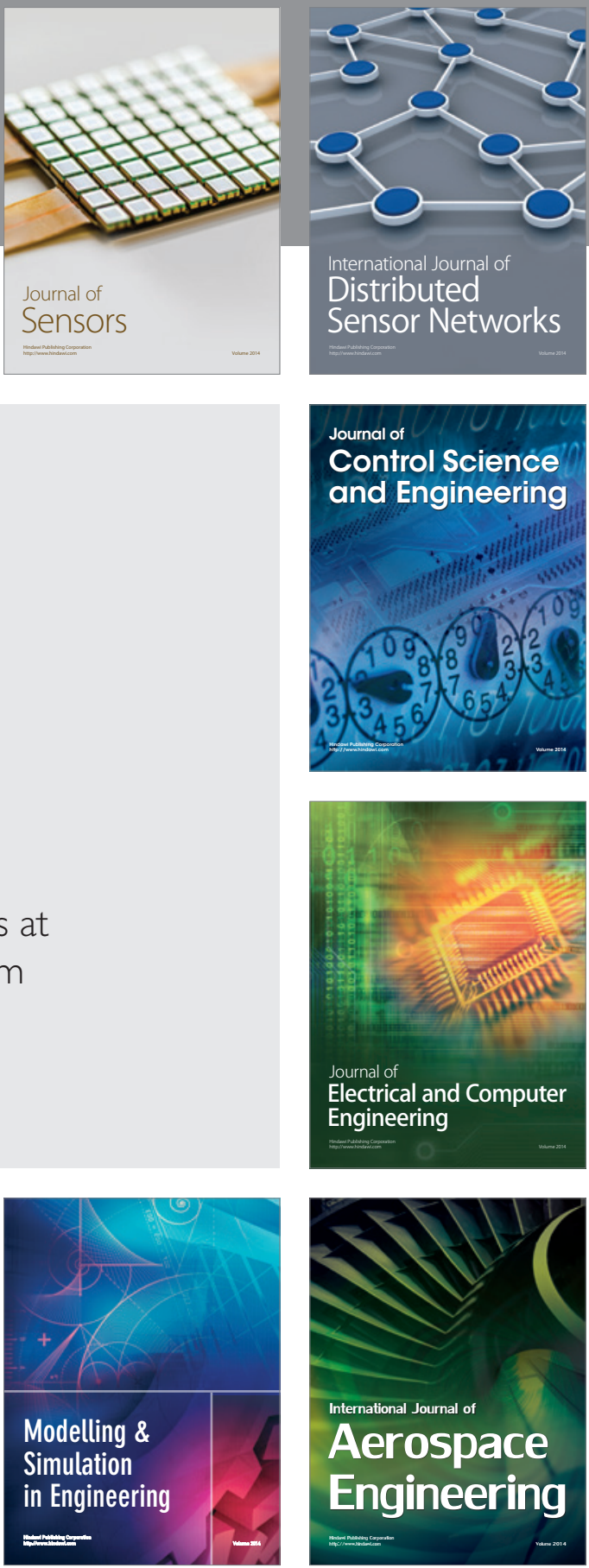

Journal of

Control Science

and Engineering
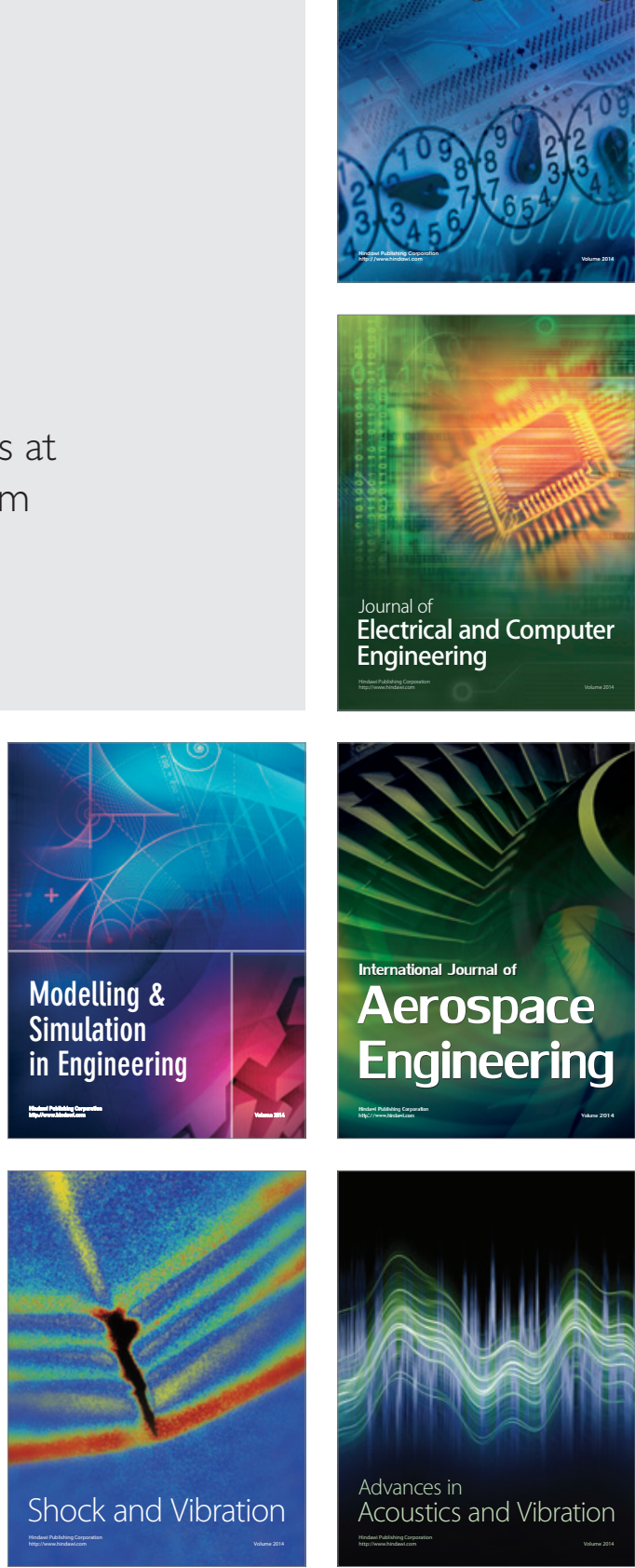